Modelling of road profiles using roughness indicators

Pär Johannesson and Igor Rychlik

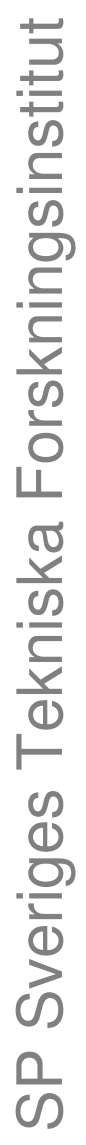




\section{Modelling of road profiles using roughness indicators}

Pär Johannesson and Igor Rychlik

SP Sveriges Tekniska Forskningsinstitut

SP Technical Research Institute of Sweden

SP Rapport 2012:43

ISBN 978-91-87017-61-2

ISSN 0284-5172

Borås 2012 


\title{
Modelling of road profiles using roughness indicators
}

\author{
PÄR JOHANNESSON* AND IGOR RYCHLIK** \\ Addresses: \\ * SP Technical Research Institute of Sweden, SE-400 22 Göteborg, Sweden (Corresponding author) \\ Par.Johannesson@sp.se \\ ** Mathematical Sciences, Chalmers University of Technology, SE-412 96 Göteborg, Sweden \\ rychlik@chalmers.se
}

\begin{abstract}
The vertical road input is the most important load for durability assessments of vehicles. We focus on stochastic modelling of the road profile with the aim to find a simple by still useful model. The proposed non-stationary Laplace model with ISO spectrum has only two parameters, and can be efficiently estimated from a sequence of roughness indicators, such as IRI or ISO roughness coefficient. Thus, a road profile can be stochastically reconstructed from roughness indicators. Further, explicit approximations for the fatigue damage due to Laplace roads are developed. The usefulness of the proposed Laplace-ISO model is validated for eight measured road profiles.
\end{abstract}

Keywords: Road surface profile, road roughness, road irregularity, Laplace process, nonGaussian process, power spectral density (PSD), ISO spectrum, roughness coefficient, international roughness index (IRI), vehicle durability, fatigue damage.

\section{Introduction}

Durability assessment of vehicle components often requires a customer or market specific load description. It is therefore desirable to have a model of the load environment that is vehicle independent and which may include many factors, such as encountered road roughness, hilliness, curvature, cargo loading, driver behaviour and legislation. Here we are concerned with modelling of the road surface roughness with focus on fatigue life prediction. Especially, we focus on reconstruction of road profiles based on measurements of the so-called International Roughness Index (IRI), which is often available from road administration data bases.

Traditionally, road profiles have been modelled by using Gaussian processes, see e.g. (Dodds and Robson, 1973; ISO 8608, 1995; Andrén, 2006). However, it is well known that measured road profiles contain shorter segments with above average irregularity, which is a property that can not be modelled by a Gaussian process, and therefore several approaches has been suggested, see e.g. (Bogsiö, 2007) and the references therein. In (Bogsiö et al., 2012) a new class of random processes, namely Laplace processes, has been proposed for modelling road profiles. Simply speaking it is a Gaussian process where the variance is randomly changing. A similar approach has been taken by (Bruscella et al., 1999; Rouillard, 2004, 2009).

In the case when only IRI data available, a simple enough model is required in order to be able to estimate the model parameters. Therefore, we will use the non-stationary Laplace model presented in (Bogsiö et al., 2012), together with the standardized spectrum according to (ISO 8608, 1995), which gives a Laplace model with only two parameters to estimate. We will demonstrate how to efficiently estimate the Laplace parameters, where the first parameter describes the mean roughness, while the second parameter describes the variability of the 
variance which is the gamma distributed. In the non-stationary Laplace model the variance is constant for short segments of fixed length (typically one or some hundred metres). We will develop a simple but accurate approximation of the fatigue damage due to Laplace road profiles. The last part is devoted to validating the model using measure road profiles.

\section{List of abbreviations}

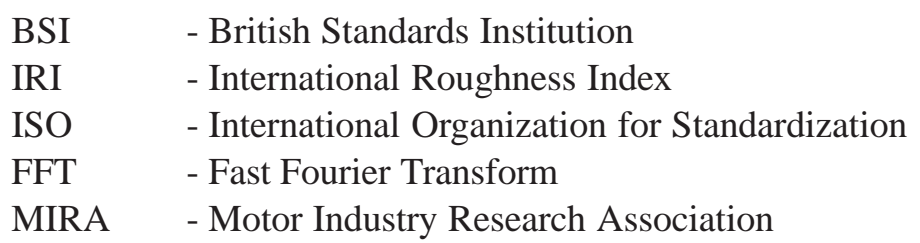

\section{List of symbols and notation}

\begin{tabular}{|c|c|}
\hline$C$ & - International Roughness Index $\left[\mathrm{m}^{3}\right]$ \\
\hline IRI & - International Roughness Index $[\mathrm{mm} / \mathrm{m}]$ \\
\hline$x$ & - position of a vehicle $[\mathrm{m}]$ \\
\hline$v$ & - vehicle speed $[\mathrm{m} / \mathrm{s}]$ \\
\hline$Z(x)$ & - road profile model [m] \\
\hline$L$ & - length of road segments $[\mathrm{m}]$ \\
\hline$L_{p}$ & - length of a road profile [m] \\
\hline$\tilde{Z}(x)$ & - normalized road profile model [-] \\
\hline$Y_{\mathrm{IRI}}(x)$ & - IRI-response of a vehicle [m] \\
\hline$\omega$ & - angular frequency $[\mathrm{rad} / \mathrm{s}]$ \\
\hline$\Omega$ & - spatial angular frequency [rad/m] \\
\hline$S_{Z}(\Omega)$ & - road profile model spectrum $\left[\mathrm{m}^{3}\right]$ \\
\hline$S_{0}(\Omega)$ & - normalized road profile model spectrum $[\mathrm{m}]$ \\
\hline$S_{Y}(\Omega)$ & - spectrum of vehicle force response $\left[\mathrm{m}^{3}\right]$ \\
\hline$S_{Y_{\text {IRI }}}(\Omega)$ & - spectrum of vehicle IRI-response $\left[\mathrm{m}^{3}\right]$ \\
\hline$H_{v}(\Omega)$ & - transfer function of force response filter at speed $v$ \\
\hline$H_{\text {IRI }, v}(\Omega)$ & - transfer function of IRI-response filter at speed $v$ \\
\hline$g(x)$ & - kernel for moving averages $\left[\mathrm{m}^{1 / 2}\right]$ \\
\hline $\mathcal{F}$ & - Fourier transform \\
\hline $\mathrm{E}[X]$ & - expectation of random variable $\mathrm{X}$ \\
\hline $\mathrm{V}[X]$ & - variance of random variable $\mathrm{X}$ \\
\hline$\sigma^{2}$ & - variance of road profile $\left[\mathrm{m}^{2}\right]$ \\
\hline$\kappa$ & - kurtosis of road profile \\
\hline & - shape parameter in Laplace models \\
\hline
\end{tabular}

\section{Road spectra and roughness coefficient}

For stationary loads, power spectra is often used to describe the energy of harmonics that build a signal. The vertical road variability consists of the slowly changing landscape (topography), the road surface unevenness (road roughness), and the high variability components (road texture). For fatigue applications, the road roughness is the relevant part of the spectrum. Often one assumes that the energy for frequencies $<0.01 \mathrm{~m}^{-1}$ (wavelengths above 100 metres) represents landscape variability, which does not affect the vehicle dynamics and hence can be 
removed from the spectrum. Similarly high frequencies $>10 \mathrm{~m}^{-1}$ (wavelengths below 10 $\mathrm{cm}$ ) are filtered out by the tire and thus are not included in the spectrum.

The ISO 8608 standard (ISO 8608, 1995) uses a two parameter spectrum to describe the road profile $Z(x)$

$$
S_{Z}(\Omega)=C\left(\frac{\Omega}{\Omega_{0}}\right)^{-w}, \quad 2 \pi \cdot 0.011 \leq \Omega \leq 2 \pi \cdot 2.83 \mathrm{rad} / \mathrm{m}, \quad \text { and zero otherwise },
$$

where $\Omega$ is the spatial angular frequency, and $\Omega_{0}=1 \mathrm{rad} / \mathrm{m}$. The spectrum is parameterized by the degree of unevenness $C$, here called the roughness coefficient, and the waviness $w$. The ISO spectrum is often used for quite short road section (in the order of 100 metres). For road classification the ISO standard uses a fixed waviness $w=2$. This simplified ISO spectrum has only one parameter, the roughness coefficient $C$. The ISO standard and classification of roads have been discussed by many authors, e.g. recently in (González et al., 2008; Ngwangwa et al., 2010).

The simplicity of the ISO spectrum makes it attractive to use in vehicle development. However, often the spectrum parameterized as in ISO 8608 does not provide an accurate description of real road spectra, and therefore many different parameterizations have been proposed, see e.g. (Andrén, 2006) where several spectral densities $S_{Z}(\Omega)$ modelings road profiles were compared.

(a)

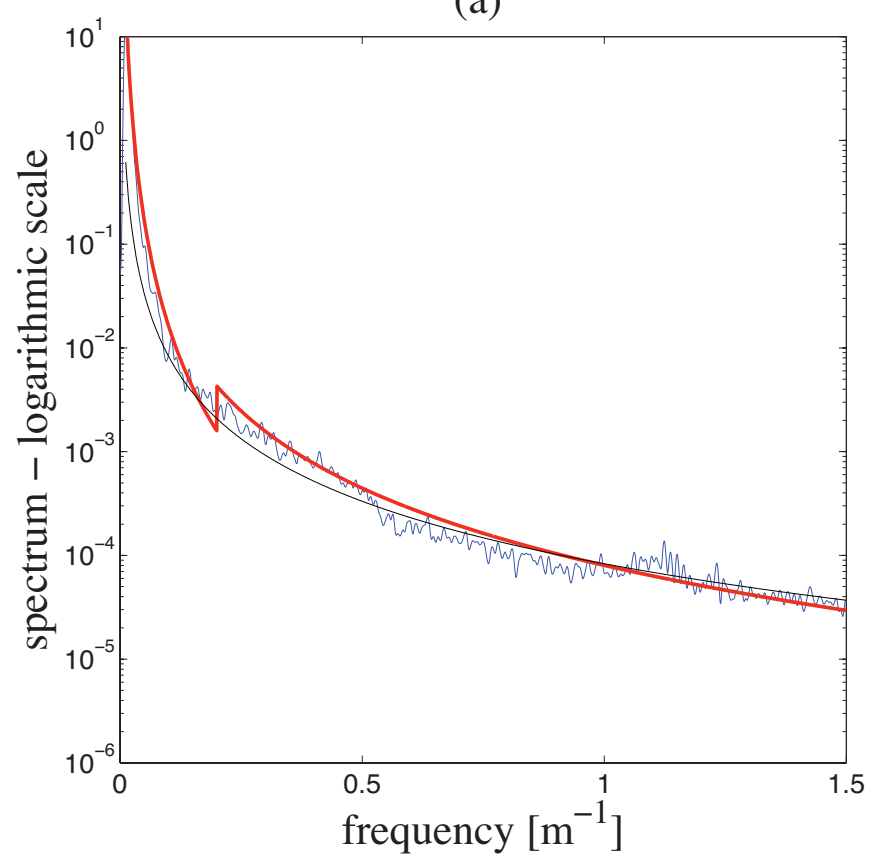

Figure 1: An example of estimated spectra for an observed normalized road profile, showing the observed spectrum (irregular line) versus the parametric estimates of ISO (thin line) and MIRA spectra (thick line).

In Figure 1, the observed spectrum (irregular line) based on a $5 \mathrm{~km}$ long road segment is compared with the fitted ISO 8608 spectrum (thin line). One can see that a single slope spectrum does not accurately describe the observed spectrum for both low and high frequencies. Therefore, a two slope spectrum, known as the MIRA (Motor Industry Research Association) 
spectra, is also employed and considered as another industry standard, see (La Barre et al., 1969). The fitted MIRA spectrum is also shown in Figure 1 (thick line), with estimated $w_{1}=3.71$ for low frequencies and $w_{2}=2.27$ for high frequencies, and fits much better to the observed spectrum than the simpler ISO spectrum.

Note that the simple parametric spectral densities will not accurately approximate the road roughness spectrum for whole range frequencies, however, what is important is that they correctly estimate the energy for frequencies in the range which may excite the vehicle response, which obviously also depends on the vehicle speed. In the present paper the ISO spectrum will be used. The choice of the ISO spectra is dictated by its simplicity, as it depends on only one parameter, which makes it easier to use in classification of large sets of diverse road profiles. Further, the parameter can be related to IRI, as will be explained below.

\section{International roughness index}

When monitoring road quality, segments of measured longitudinal road profiles are often condensed into a sequence of IRI values, see (Gillespie et al., 1986). They are calculated using a quarter-car vehicle model, see Figure 2, whose response at speed $80 \mathrm{~km} / \mathrm{h}$ is accumulated to yield a roughness index with units of slope (in/mi, m/ $\mathrm{km}$, etc.). Since its introduction in 1986, IRI has become the road roughness index most commonly used worldwide for evaluating and managing road systems.

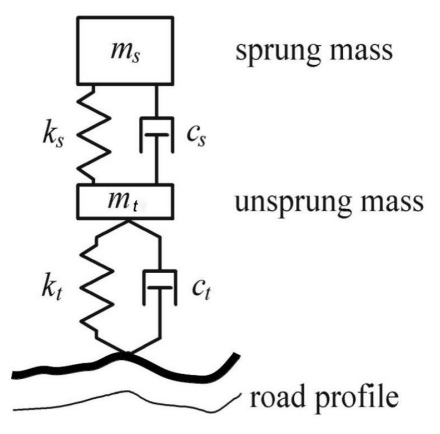

Figure 2: Quarter vehicle model.

More precisely, IRI is defined as the accumulated suspension motion divided by the distance travelled. The parameters of the quarter vehicle is defined by the so-called Golden Car with parameters given in Table1. The response is the difference between motions of the sprung and unsprung masses, denoted by $Y_{\mathrm{IRI}}(x)=X_{s}(x)-X_{u}(s)$. This defines a filter of the road profile, which at speed $v$ has the following transfer function

$$
H_{\text {IRI }, v}(\Omega)=\frac{-\omega^{2} k_{1}}{\left(k_{2}+i \omega c\right)\left(k_{2}+k_{1}+i \omega c-\omega^{2} \mu\right)-\left(k_{2}+i \omega c\right)},
$$

where $\omega=\Omega \cdot v$ is the angular frequency having units $\mathrm{rad} / \mathrm{s}$. For a road segment of length $L$, the IRI can be expressed as the average total variation of $Y(x)$, viz.

$$
\mathrm{IRI}=1000 \frac{1}{v L} \int_{0}^{L}\left|\dot{Y}_{\mathrm{IRI}}(x)\right| \mathrm{d} x
$$

with speed $v=80 \mathrm{~km} / \mathrm{h}=22.22 \mathrm{~m} / \mathrm{s}$. The factor 1000 appears since IRI has units $\mathrm{mm} / \mathrm{m}$ and $Y_{\mathrm{IRI}}(x)$ is in metres. Thus, $1000 \cdot \dot{Y}_{\mathrm{IRI}}(x)$ is the relative suspension speed in unit $\mathrm{mm} / \mathrm{s}$ 
computed at location $x$. More details on quarter vehicle modelling can be found in e.g. (Howe et al., 2004).

Table 1: Parameters of quarter vehicle models.

\begin{tabular}{lrl}
\multicolumn{3}{c}{ Golden Car } \\
\hline Symbol & Value & Unit \\
\hline$c=c_{s} / m_{s}$ & 6.0 & $\mathrm{~s}^{-1}$ \\
$k_{1}=k_{t} / m_{s}$ & 653 & $\mathrm{~s}^{-2}$ \\
$k_{2}=k_{s} / m_{s}$ & 63.3 & $\mathrm{~s}^{-2}$ \\
$\mu=m_{u} / m_{s}$ & 0.15 & - \\
\hline
\end{tabular}

\begin{tabular}{lrl}
\multicolumn{3}{c}{ Quarter Truck } \\
\hline Symbol & Value & Unit \\
\hline$m_{s}$ & 3400 & $\mathrm{~kg}$ \\
$k_{s}$ & 270000 & $\mathrm{~N} / \mathrm{m}$ \\
$c_{s}$ & 6000 & $\mathrm{Ns} / \mathrm{m}$ \\
$m_{t}$ & 350 & $\mathrm{~kg}$ \\
$k_{t}$ & 950000 & $\mathrm{~N} / \mathrm{m}$ \\
$c_{t}$ & 300 & $\mathrm{Ns} / \mathrm{m}$ \\
\hline
\end{tabular}

Next, we will compute IRI, for a Gaussian road model with ISO spectrum $S_{Z}(\Omega)$, see Eq. (1). The response $Y_{\text {IRI }}(x)$, for the "Golden Car" has power spectral density given by

$$
S_{Y_{\text {IRI }}}(\Omega)=\left|H_{\text {IRI }, v}(\Omega)\right|^{2} S_{Z}(\Omega) .
$$

Assuming a Gaussian model for road profile, the expected IRI can then be computed as

$$
\mathrm{E}[\mathrm{IRI}]=\mathrm{E}\left[1000 \frac{1}{v L} \int_{0}^{L}\left|\dot{Y}_{\mathrm{IRI}}(x)\right| \mathrm{d} x\right]=\frac{1000}{v} \mathrm{E}\left[\left|\dot{Y}_{\mathrm{IRI}}(0)\right|\right]=\frac{1000}{v} \cdot \sqrt{\frac{2 \lambda_{2}}{\pi}},
$$

where $\lambda_{i}$ is the $i$ :th spectral moment of the response

$$
\lambda_{i}=\int_{0}^{\infty} \Omega^{i} S_{Y_{\mathrm{IRI}}}(\Omega) d \Omega .
$$

It can be shown that the expected "theoretical" IRI can be expressed as

$$
\mathrm{E}[\mathrm{IRI}]=A(w, v) \cdot \sqrt{C},
$$

where $A(w, v)$ is a constant depending on the waviness $w$ and the speed $v$. For the Golden car and ISO spectrum with waviness $w=2$, the formula simplifies to

$$
\mathrm{E}[\mathrm{IRI}]=2.21 \cdot \sqrt{C},
$$

where the roughness coefficient $C$ has units $\mathrm{m} \cdot \mathrm{mm}^{2}$. This theoretically derived relation between IRI and $C$ agrees with the empirically formula by (Kropáč and Múčka, 2004, 2007).

Denote by $\hat{I}$ an estimate of $\mathrm{E}[\mathrm{IRI}]$, e.g. the average of observed IRI. In this paper we will use Eq. (5) where $\lambda_{2}$ will be estimated from the observed power spectral density, see Eq. (6), or by estimating variance of the $\dot{Y}$. The roughness coefficient $C$ will then be estimated from IRI by

$$
\hat{C}=\left(\frac{\hat{I}}{2.21}\right)^{2} .
$$

\section{Fatigue Damage Index}

We will here define a fatigue damage index $D_{v}(k)$ that is assessed by studying the response of a quarter-vehicle model travelling at a constant speed on road profiles, see Figure 2 To be more precise, the response considered is the force acting on the sprung mass $m_{s}$. Such 
a simplification of a physical vehicle cannot be expected to predict loads exactly, but it will highlight the most important road characteristics as far as fatigue damage accumulation is concerned. The parameters in the model are set to mimic heavy vehicle dynamics, following (Bogsiö, 2007). Thus, the values of the parameters differ somewhat form the ones defining the Golden car, see Table 1

Neglecting possible "jumps", which occur when a vehicle looses contact with the road surface, the response of the quarter-vehicle, i.e. the force $Y(x)=m_{s} \ddot{X}_{s}(x)$, as a function of vehicle location $x$, can be computed through linear filtering of the road profile. The filter at speed $v$ has the following transfer function

$$
H_{v}(\Omega)=\frac{m_{s} \omega^{2}\left(k_{t}+i \omega c_{t}\right)}{k_{t}-\frac{\left(k_{s}+i \omega c_{s}\right) \omega^{2} m_{s}}{-m_{s} \omega^{2}+k_{s}+i \omega c_{s}}-m_{t} \omega^{2}+i \omega c_{t}}\left(1+\frac{m_{s} \omega^{2}}{k_{s}-m_{s} \omega^{2}+i \omega c_{s}}\right),
$$

where $\omega=\Omega \cdot v$ is the angular frequency having units $\mathrm{rad} / \mathrm{s}$.

For a stationary road model $Z(x)$ having power spectral density $S_{Z}(\Omega)$, the response $Y(x)$, for a vehicle at speed $v[\mathrm{~m} / \mathrm{s}]$, has power spectral density given by

$$
S_{Y}(\Omega)=\left|H_{v}(\Omega)\right|^{2} S_{Z}(\Omega), \quad S_{Z}(\Omega)=\sigma^{2} S_{0}(\Omega),
$$

where $\sigma^{2}=\int_{-\infty}^{\infty} S_{Z}(\Omega) d \Omega$. Note that $\sigma^{2}$ is a variance of the road profile model and it may not be equal to the measured road profile variance, e.g. when $S_{Z}(\Omega)$ is ISO spectrum.

In general the response $Y(x)$, which is the force acting on the sprung mass, is computed by means of filtering the signal $Z(x)$ using the filter with transfer function $H_{v}(\Omega)$ given in Eq. (10), which depends on the vehicle speed $v$. In the example $v=10,15[\mathrm{~m} / \mathrm{s}]$ have been used. The response of the quarter vehicle $Y(x)$ is the solution of a fourth order ordinary differential equation or alternatively a convolution of $Z(x)$ with the vehicle's impulse response $h_{v}(x)$, viz.

$$
Y(x)=\int_{-\infty}^{x} h_{v}(x-u) Z(u) \mathrm{d} u .
$$

In this paper responses for measured and simulated roads are computed using the FFT algorithm. Since the initial conditions of the system at $t=0$ are unknown the Hanning window has been used to make the start and the end of the ride smooth. This is necessary or otherwise the first oscillation of the response may cause all the damage - the car is hitting a wall.

The purpose of this work is to propose models for $Z(x)$ defined by means of few parameters that could be used to compute $Y(x)$ or other more complex and realistic responses in such a way that the risk for fatigue failure, or extremal responses, could be quantified. Hence the most important criterion for a good model of a measured road profile is that the rainflow damage of the response is well represented.

The rainflow damage is computed in two steps. First rainflow ranges $\Delta S_{\mathrm{rfc}, i}$ in the load $Y(x), 0 \leq x \leq L_{p}$, are found, then the rainflow damage per metre is computed according to Palmgren-Miner rule (Palmgren, 1924; Miner, 1945), viz.

$$
D_{v}(k)=\frac{1}{L_{p}} \sum_{i} \Delta S_{\mathrm{rfc}, i}^{k}
$$

see also (Rychlik, 1987) for details of this approach. In this paper $2 \leq k \leq 5$, have been used. The damage $D_{v}(k)$ for higher exponent value $k=5$ depends mostly on the proportion and size of large cycles, while damage for $k=3$, corresponding to the crack growth process, 
depends on the sizes of both large and moderately large cycles. For a stationary load, $D_{v}(k)$ converges to a limit as $L_{p}$ increases without bounds. However, for short road profiles, $D_{v}(k)$ may vary considerably. For ergodic loads the limit is equal to the expected damage $\mathrm{E}\left[D_{v}(k)\right]$. In Section 6, computations of the expected damage will be further discussed. In these computations, the response to the normalized road profile $\tilde{Z}(x)$ having the spectrum $S_{0}(\Omega)$ will be employed, viz.

$$
\tilde{Y}(x)=\int_{-\infty}^{x} h_{v}(x-u) \tilde{Z}(u) \mathrm{d} u .
$$

The spectrum of $\tilde{Y}(x)$ is given by

$$
S_{\tilde{Y}}(\Omega)=\left|H_{v}(\Omega)\right|^{2} S_{0}(\Omega) .
$$

\section{Stochastic models for road profiles}

Parts of this section follows (Bogsiö et al., 2012). First, the commonly used stationary Gaussian model will be presented. Then, in Section 5.2, we introduce the non-stationary Gaussian model with variable variances between short sections, but with smooth transitions between the segments, and then extend it to the non-stationary Laplace model where the variable variance is modelled by a Gamma distribution. Recall that, for a road profile $Z(x)$ with standard deviation $\sigma$, we denote by $\tilde{Z}(x)$ the normalized profile, i.e. $\mathrm{E}[\tilde{Z}(x)]=0$ and $\mathrm{V}[\tilde{Z}(x)]=1$. Thus, for a zero mean profile, $Z(x)=\sigma \tilde{Z}(x)$ with spectrum $S_{Z}(\Omega)=\sigma^{2} S_{0}(\Omega)$, where $S_{0}(\Omega)$ is the spectrum of the normalized road profile $\tilde{Z}(x)$

In the following sections we will discuss Laplace models with ISO spectrum and give means to estimate parameters in the model from an observed IRI sequence. Note that the IRI is often available in road maintenance databases. MATLAB code to simulate the road models is given in Appendix B

\subsection{Stationary Gaussian model}

A zero mean stationary Gaussian process is completely defined by its mean and power spectrum, thus, any probability statement about properties of Gaussian processes can in principle be expressed by means of the spectrum. This is not always practically possible and hence Monte Carlo methods are often employed. There are several ways to generate Gaussian sample paths. The algorithm proposed in (Shinozuka, 1971) is often used in engineering. It is based on the spectral representation of a stationary process. Here we use an alternative way that expresses a Gaussian process as a moving average of white noise.

Roughly speaking a moving average process is a convolution of a kernel function $g(x)$, say, with a infinitesimal "white noise" process having variance equal to the spatial discretization step, say $\mathrm{d} x$. Consider a kernel function $g(x)$, which is normalized so that its square integrates to one. Then the standardized Gaussian process can be approximated by

$$
\tilde{Z}(x) \approx \sum g\left(x-x_{i}\right) Z_{i} \sqrt{\mathrm{d} x},
$$

where the $Z_{i}$ 's are independent standard Gaussian random variables, while $\mathrm{d} x$ is the discretization step, here reciprocal of the sampling frequency $(\mathrm{d} x=5 \mathrm{~cm})$. An appropriate choice of the length of the increment $\mathrm{d} x$ is related to smoothness of the kernel.

In order to get a Gaussian process with a desired spectral density one has to use an appropriate kernel $g(x)$. Consider a symmetric kernel, i.e. $g(-x)=g(x)$. In this case, the spectrum 
$S_{0}(\Omega)$ of $\tilde{Z}(x)$ uniquely defines the kernel $g(x)$ since

$$
S_{0}(\Omega)=\frac{1}{2 \pi}|\mathcal{F} g(\Omega)|^{2},
$$

where $\mathcal{F} g(\Omega)$ stands for the Fourier transform, and for symmetric kernels their Fourier transform is given by

$$
\mathcal{F} g(\Omega)=\sqrt{2 \pi S_{0}(\Omega)}
$$

\subsection{Non-stationary models}

Stationary Gaussian loads have been extensively studied in literature and applied as models for road roughness, see e.g. (Dodds and Robson, 1973) for an early application. However, the authors of that paper were aware that Gaussian processes cannot "exactly reproduce the profile of a real road". In (Charles, 1993) a non-stationary model was proposed, constructed as a sequence of independent Gaussian processes of varying standard deviations but the same standardized spectrum $S_{0}(\Omega)$. Knowing durations and sizes of standard deviations the model is a non-stationary Gaussian process. Similar approaches were used in (Bruscella et al., 1999; Rouillard, 2004, 2009). The variability of the standard deviation $\sigma$ was modelled by a discrete distribution taking a few number of values (in published work the number of values was six). In (Rouillard, 2009) random lengths of constant variance sections were also considered. In those papers one was not concerned with the problem of connecting the segments with constant variances into one signal since the response was modelled as a non-stationary Gaussian process, i.e. by a process of the same type as the model of the road surface. Such individual treatment of the constant variance segments is possible only if they are much longer than the support of the kernel $g(x)$, e.g. in the order of kilometres. However, actual roads contain much shorter sections with above-average irregularity. These irregularities cause most of the vehicle fatigue damage, as reported in (Bogsiö, 2007).

Since we are dealing with non-stationary models it is not obvious how the normalized road profile $\tilde{Z}(x)$ should be defined. Here we will assume that $\tilde{Z}(x), x \in\left[0, L_{p}\right]$ has mean zero and variance one, which means that the mean and variance of $\tilde{Z}(x)$ at a point $x$ chosen at random from $\left[0, L_{p}\right]$ are zero and one, respectively.

For example suppose that the normalized road profile $\tilde{Z}(x), x \in[0, L]$, consists of $M$ equally long segments of length $L=L_{p} / M$, where the constant variance of the $j$ :th segment is equal to $r_{j}, j=1, \ldots, M$ with $\frac{1}{M} \sum_{j=1}^{M} r_{j}=1$ since $\tilde{Z}(x)$ has variance one. However, such a process is discontinuous at times where the variance is changing. Although formally correct, the model induces a transient largely contributing to the fatigue damage each time a vehicle passes these locations. A more realistic approach can be made by continuous transitions between segments of constant variance. This can be done in different ways but here we employ moving averages of "non-stationary" white noise to define a smooth version of $\tilde{Z}(x)$. First we present the non-stationary Gaussian model with variable variances but smooth transitions between the segments, and then extend it to the non-stationary Laplace model.

\subsection{Non-stationary Gaussian model}

The process consists of $M$ segments of length $L=L_{p} / M$ and we wish to define a process on $\left[0, L_{p}\right]$. We would like that each segment has a prescribed standard deviation $\sigma_{j}, j=$ $1, \ldots, M$. Obviously the variance of the process is $\sigma^{2}=\frac{1}{M} \sum_{j=1}^{M} \sigma_{j}^{2}$. 
Denote the standardized variance of the $j$ :th segment by

$$
r_{j}=\sigma_{j}^{2} / \sigma^{2}
$$

Next we will define the $j$ :th non-stationary Gaussian process $\tilde{Z}_{j}(x)$ process for all $0 \leq x \leq$ $L_{p}$. Let again $\mathrm{d} x$ be the sampling step of the process and $\left[s_{j-1}, s_{j}\right], s_{j}-s_{j-1}=L$, the interval where the road profile model would have the variance $\sigma_{j}^{2}$, viz. $s_{0}=0<s_{1}<\ldots<s_{M}=L_{p}$. Now define $M$ processes $\tilde{Z}_{j}(x)$ as follows

$$
\tilde{Z}_{j}(x) \approx \sum_{s_{j-1}<x_{i} \leq s_{j}} g\left(x-x_{i}\right) \sqrt{r_{j}} Z_{i} \sqrt{\mathrm{d} x}
$$

where the $Z_{i}$ 's are independent standard Gaussian variables, and $\mathrm{d} x$ is the discretization step. Finally the road profile model $Z(x)$ is given by

$$
Z(x)=\sigma \tilde{Z}(x)=\sigma \sum_{j=1}^{M} \tilde{Z}_{j}(x) .
$$

\subsection{Non-stationary Laplace model}

A reasonable length of road segments with constant variance is 200 metres. This would mean that in order to describe a $10 \mathrm{~km}$ long road with ISO spectrum one would need $50 \sigma_{j}^{2}$ parameters. This is not very convenient and therefore in (Bogsiö et al., 2012) another approach was taken. Namely, the variability of the standardized variances $r_{j}$, defined in Eq. (19), was modelled by means of the Gamma probability distribution, i.e. $r_{j}$ are independent observations of a random variable $R$ having probability distribution function

$$
f_{R}(r)=\frac{\theta^{\theta}}{\Gamma(\theta)} r^{\theta-1} \exp (-r \theta)
$$

By replacing $r_{j}$ in Eq. (20) by independent Gamma random variables $R_{j}$, we get

$$
\tilde{Z}_{j}(x) \approx \sum_{s_{j-1}<x_{i} \leq s_{j}} g\left(x-x_{i}\right) \sqrt{R_{j}} Z_{i} \sqrt{\mathrm{d} x} .
$$

and the road profile $Z(x)$ is given by

$$
Z(x)=\sigma \tilde{Z}(x)=\sigma \sum_{j=1}^{M} \tilde{Z}_{j}(x) .
$$

Further, for a zero mean Gaussian random variable $Z_{j}$, the product $\sqrt{R_{j}} Z_{j}$ has a Laplace distribution, and thus the process $Z(x)$, defined in Eq. (24), has a generalized Laplace distribution, see (Kotz et al., 2001). MATLAB code to simulate this model can be found in Appendix B

It should be noted that a non-stationary Laplace road model requires only two parameters; the variance $\sigma^{2}$ of the process and the shape parameter $\theta$ of the Gamma distribution. In Laplace modelling, traditionally another parameterization is used, viz. $\nu=1 / \theta$. Now if the shape parameter $\nu$ is close to zero then the Laplace process is close to be a Gaussian process. The shape parameter $\nu$ of the Laplace process can also be computed from the kurtosis $\kappa$ of the process $Z(x)$, namely

$$
\nu=(\kappa-3) / 3 .
$$


Alternatively, if the variances of the road segments with constant variance are known, then the moment method gives the following estimates

$$
\hat{\sigma}^{2}=\frac{1}{M} \sum_{j=1}^{M} \hat{\sigma}_{j}^{2}, \quad \hat{\nu}=\frac{\frac{1}{M-1} \sum_{j=1}^{M}\left(\hat{\sigma}_{j}^{2}-\hat{\sigma}^{2}\right)^{2}}{\left(\hat{\sigma}^{2}\right)^{2}} .
$$

\subsection{Road models with ISO spectrum}

The kernel $g(x)$ defined by the ISO spectrum, which in the standardized form (variance one and waviness $w=2$ ) is given by

$$
S_{0}(\Omega)=C_{0}\left(\frac{\Omega}{\Omega_{0}}\right)^{-2}, \quad C_{0}=14.4 \mathrm{~m}^{3}, \quad 2 \pi \cdot 0.011 \leq \Omega \leq 2 \pi \cdot 2.83 \mathrm{rad} / \mathrm{m},
$$

and zero otherwise. Recall that $\Omega_{0}=1 \mathrm{rad} / \mathrm{m}$. The stationary Gaussian model with ISO spectrum has only one parameter, the roughness coefficient $C$, or alternatively the variance $\sigma^{2}=C / C_{0}$. It is important to notice that $\sigma^{2}$ is usually smaller than the variance of the measured road elevation, since it is chosen in such a way that the "true" spectrum is well approximated at the frequency range of interest. In Figure 3 the symmetrical kernel defined by the ISO spectrum is presented.

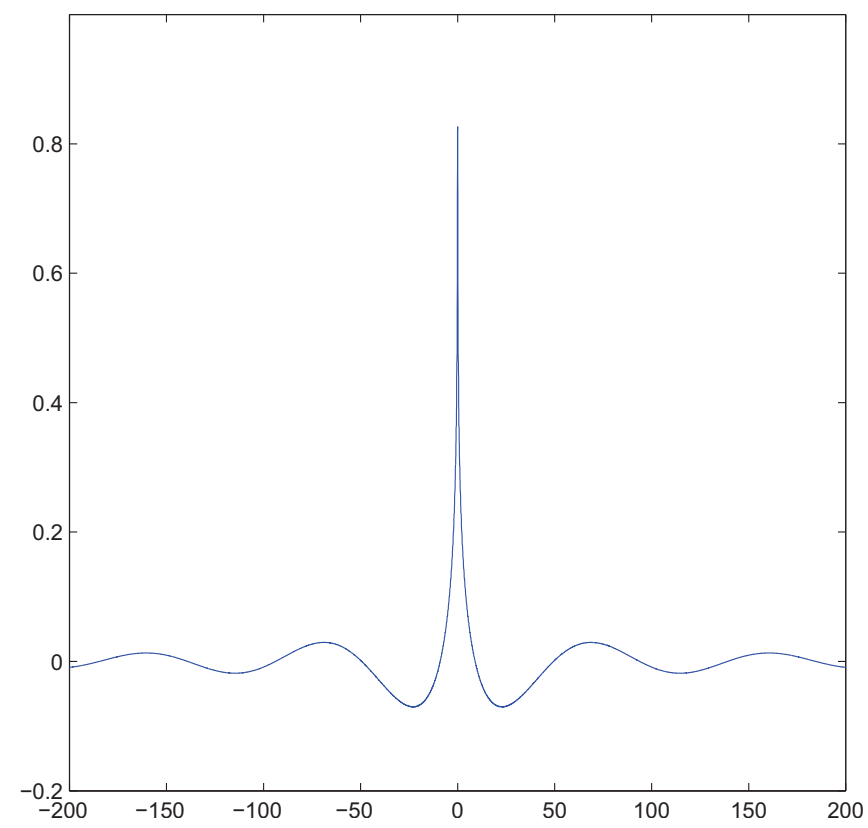

Figure 3: ISO kernel $g(x)$.

In order to define the non-stationary Gaussian model one need determine $L$, the length of segments of constant variance, and then estimate the mean roughness $C$ as well as the sequence of relative variances $r_{j}$. Often a suitable value of $L$ is chosen by experience, typically $L=200$ $\mathrm{m}$, while $C$ and the $r_{j}$ 's can be estimated from a sequence of IRI values by means of Eq. (9). The procedure results in a large number parameters and therefore we propose to describe the variability of $r_{j}$ by means a stochastic model. If the $r_{j}$ 's are obtained as independent values from a gamma distribution then $Z(x)$ is called Laplace road surface model with gamma variance of segments. 
In order to define the Laplace model with ISO spectrum, we need two parameters, the mean roughness $C$ and the Laplace shape parameter $\nu$, modelling the gamma variances for the segments of length $L$. Consequently, by introducing one additional parameter $\nu$, the stationary Gaussian model is extended to the non-stationary Laplace model. Recall that setting the parameter $\nu$ equal zero gives the stationary Gaussian case.

\subsection{Estimation of Laplace road models with ISO spectrum}

How to estimate the parameters in a Laplace, or a Gaussian model, with ISO spectrum is not obvious and many possible approaches are possible. The difficulty lies in the fact that an "useful" ISO model has variance $\sigma^{2}$ and kurtosis $\kappa$ that differ from the variance and kurtosis estimated from measured road profile. Consequently, relations (25) 26 and $C=\sigma^{2} C_{0}$ are no appropriate estimators when $\hat{\sigma}_{j}^{2}$ are estimated variances from measured road profiles.

However, Eq. 26) is still useful if the $\hat{\sigma}_{j}^{2}$ 's are replaced by roughness coefficients $\hat{C}_{j}$ 's, defining ISO spectra, for short road segments. The roughness coefficients $C_{j}$ could be estimated by means of some statistical procedure if the measured profile is available. However, this is seldom the case, and hence we will propose to estimate $C_{j}$ from a sequence of IRI values using Eq. (8). Note that IRI parameters are often available from road databases maintained by road agencies.

Summarizing, we propose to estimate $C_{j}$ by

$$
\hat{C}_{j}=\left(\frac{\hat{I}_{j}}{2.21}\right)^{2}
$$

where $\hat{I}_{j}$ is an estimate of IRI of the $j$ :th segment, see Eq. (3). Having a sequence of estimates $\hat{C}_{j}, j=1, \ldots, M$, it is possible to estimate $C$ by the average of $\hat{C}_{j}$. Next, since $C_{j}$ is proportional to the variance $\sigma_{j}^{2}$, for $Z(x)$ with ISO spectrum, one can also use $\hat{C}_{j} / \hat{C}$ as estimates of $r_{j}$ and, for example, the maximum likelihood method can be used to estimate the shape parameter $\nu$. However, for simplicity of presentation, the moment method will be employed here, giving the following estimates

$$
\hat{C}=\frac{1}{M} \sum_{j=1}^{M} \hat{C}_{j}, \quad \hat{\nu}=\frac{\frac{1}{M-1} \sum_{j=1}^{M}\left(\hat{C}_{j}-\hat{C}\right)^{2}}{\hat{C}^{2}} .
$$

\section{Expected damage index}

The purpose of this section is to present a closed form approximation for the expected damage index for the Laplace road profile model with ISO spectrum. The important special case of Gaussian response, $\nu=0$, have been intensively studied in the literature and many approximations are available, see (Bengtsson and Rychlik, 2009) for comparisons of different approaches. Therefore, we wish to relate the expected damage index for the Laplace model to the expected Gaussian index.

For the Gaussian model, the damage index $D_{v}(k)$, defined in Eq. (13), depends on the following parameters; the speed $v$, the exponent $k$ in the S-N curve, the road roughness coefficient $C$, see Eqs. (11) and (27) for ISO spectrum. For the Laplace model, the damage index depends additionally on the shape parameter $\nu$. In order to make the dependence explicit in the notation we will write $D_{v}(k, C, \nu)$ for $D_{v}(k)$. 
We turn next to the main result of this section the approximation of $\mathrm{E}\left[D_{v}(k, C, \nu)\right]$. The approximation can be used for a response defined by any linear filter excited by the random Laplace road having ISO spectrum, see Eqs. (23).24). The approximation is given by

$$
\mathrm{E}\left[D_{v}(k, C, \nu)\right] \approx \mathrm{E}\left[D_{v_{0}}\left(k, C_{0}, 0\right)\right]\left(\frac{C}{C_{0}}\right)^{k / 2}\left(\frac{v}{v_{0}}\right)^{k / 2-1} \nu^{k / 2} \frac{\Gamma(k / 2+1 / \nu)}{\Gamma(1 / \nu)},
$$

where $v_{0}>0$ is a suitably chosen reference speed and $C_{0}=14.4 \mathrm{~m}^{3}$ is the roughness coefficient representing a normalized road profile, see Eq. (27). More details on the derivation of Eq. (30) is found in Appendix A. In examples we will use $v_{0}=10 \mathrm{~m} / \mathrm{s}$. Here $\mathrm{E}\left[D_{v_{0}}\left(k, C_{0}, 0\right)\right]$ is the expected damage index for a Gaussian response, see (Bengtsson and Rychlik, 2009) for means to compute the index. The approximation is derived under assumption that the road profile has ISO spectrum. It is accurate if the length of segments of constant variance $L$ is long enough so that the influence of transients caused by change of variance can be neglected. In practice we found that $L$ about 100 metres or longer is a good choice. Note that for a nonstationary Laplace model $\left(\sigma^{2} \nu\right)$ is equal to the variance of the random variances of Gaussian segments. In addition, using Stirlings formula one can demonstrate that

$$
\nu^{k / 2} \frac{\Gamma(k / 2+1 / \nu)}{\Gamma(1 / \nu)} \rightarrow 1
$$

as $\nu$ tends to zero, i.e. the Laplace model approaches the Gaussian model.

In order to get explicit algebraic approximation for the expected damage index we will employ the so-called narrow band approximation for $\mathrm{E}\left[D_{v_{0}}\left(k, C_{0}, 0\right)\right]$ introduced in (Bendat, 1964), which actually is an upper bound for the expected damage, see (Rychlik, 1993) for a proof and (Bogsiö and Rychlik, 2009) for related results. For a road profile $\tilde{Z}(x)$ modelled as a Gaussian process with standardized ISO spectrum and the quarter vehicle travelling with speed $v_{0}=10 \mathrm{~m} / \mathrm{s}$ with transfer function given by Eq. (10), the narrow band bound is given by

$$
\mathrm{E}\left[D_{v_{0}}\left(k, C_{0}, 0\right)\right] \leq 0.35\left(5.52 \cdot 10^{10}\right)^{k / 2} \Gamma(k / 2+1) 2^{3 k / 2} .
$$

By combining Eqs. (30) and (32) we obtain the following approximation

$$
\mathrm{E}\left[D_{v}(k, C, \nu)\right] \lesssim 0.35 \cdot\left(4.615 \cdot 10^{4} \cdot C\right)^{k} \Gamma(k / 2+1)\left(\frac{v}{v_{0}}\right)^{k / 2-1} \nu^{k / 2} \frac{\Gamma(k / 2+1 / \nu)}{\Gamma(1 / \nu)} .
$$

Similar formulas can be given for any transfer function $H_{v}(\Omega)$, simply the constants 0.35 and $4.61510^{4}$ need to be modified.

Finally, based on a very long simulation the following relation has been fitted

$$
\ln \left(\mathrm{E}\left[D_{v_{0}}\left(k, C_{0}, 0\right)\right]\right)=-2.646+13.92 \cdot k .
$$

In Figure 4 the stars are estimates of $\ln \left(\mathrm{E}\left[D_{v_{0}}\left(k, C_{0}, 0\right)\right]\right)$ while the solid line is the fitted regression. As can be seen in the figure, the error is negligible for $2 \leq k \leq 7$, in fact the error is less than $0.5 \%$. Note that the regression is only valid for the quarter car response, i.e. $H_{v}(\Omega)$ given in Eq. (10). For other filters the regression will be different.

Combining Eqs. (30) and (34) lead to the following approximation of the expected damage index for road with ISO spectrum having average roughness coefficient $C$ approximated by

$$
\mathrm{E}\left[D_{v}(k, C, \nu)\right] \approx 0.07093 e^{13.92 k}\left(\frac{C}{C_{0}}\right)^{k / 2}\left(\frac{v}{v_{0}}\right)^{k / 2-1} \nu^{k / 2} \frac{\Gamma(k / 2+1 / \nu)}{\Gamma(1 / \nu)},
$$

where $C_{0}=14.4 \mathrm{~m}^{3}$ and $v_{0}=10 \mathrm{~m} / \mathrm{s}$. 


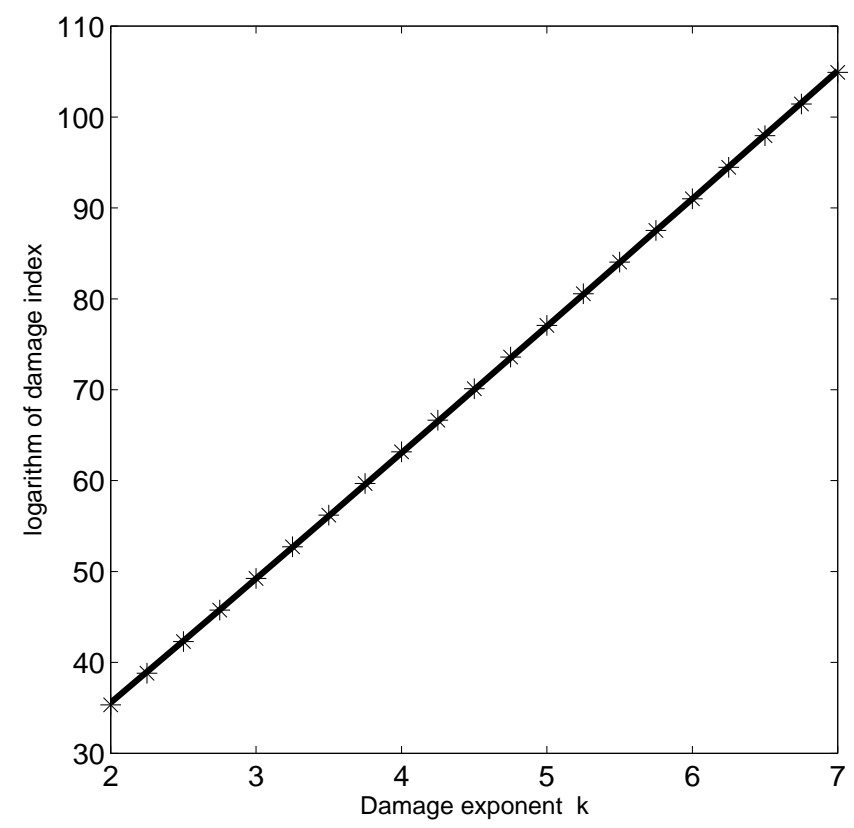

Figure 4: Stars: observed damage indices in simulated $400 \mathrm{~km}$ long Gaussian road profiles with normalized ISO spectrum, $C=C_{0}=14.4 \mathrm{~m}^{3}$, and $v=v_{0}=10 \mathrm{~m} / \mathrm{s}$. Solid line: regression line of $\ln \left(\mathrm{E}\left[D_{v_{0}}\left(k, C_{0}, 0\right)\right]\right)=-2.646+13.92 \cdot k$.

\subsection{Estimation of Laplace road models with ISO spectrum}

The relation (35) could also be used to estimate or validate parameters $C, \nu$ in Laplace-ISO road profile models, when the damage indices are available. A natural approach would be to fit relation (35) to the estimated damage indices by means of the least squares method. However, for simplicity, we will here only give explicit formulas for the estimates by inverting Eq. 35] for fixed speed $v$ and damage exponents $k=2,4$, viz.

$$
\mathrm{E}\left[D_{v}(2, C, \nu)\right] \approx 6.108 \cdot 10^{9} C, \text { while } \quad \mathrm{E}\left[D_{v}(4, C, \nu)\right] \approx 5.254 \cdot 10^{20}(\nu+1) C^{2} \frac{v}{v_{0}},
$$

which are valid for Laplace ISO road profile models. Consequently, if the model is valid and the damage indices are known then the parameters of the models can be estimated by means of Eq. (36), viz.

$$
\begin{aligned}
\hat{C} & =1.637 \cdot 10^{-10} \cdot D_{v}(2), \\
\hat{\nu} & =0.07101 \cdot \frac{v_{0}}{v} \cdot \frac{D_{v}(4)}{D_{v}(2)^{2}}-1 .
\end{aligned}
$$

\subsection{A numerical example}

In this example we will illustrate the accuracy of the approximation (30) of the expected damage index as function of the Laplace shape parameter $\nu$. Two speeds $v=10,25 \mathrm{~m} / \mathrm{s}$ and fatigue exponents $k=3,5$ will be considered. The expected damage index for a Gaussian response $\mathrm{E}\left[D_{v_{0}}(k, 1,0)\right]$ has been estimated using Eq. (13) for a $400 \mathrm{~km}$ long simulated road profile. Then approximation (30) has been used to estimate $\mathrm{E}\left[D_{v}(k, 1, \nu)\right]$. Results of the 
study is presented in Figure 5 as solid lines. In the figure we also show the narrow band approximation (upper bound), Eq. (33), as the dashed line. As expected the approximation is bounding the mean damage index. However, it is still possible to get observed indices that exceeds the bound. Finally, for each value of $\nu$, four damage indices $D_{v}(k, 1, \nu)$ have been calculated from $20 \mathrm{~km}$ long non-stationary Laplace simulations with increasing value of parameter $\nu$. The simulated indices are marked as dots for $v=25 \mathrm{~m} / \mathrm{s}$ and stars for $v=10 \mathrm{~m} / \mathrm{s}$. The agreement between the approximation of expected damage index given in Eq. (30) and the simulated indices is striking. For high values of the Laplace shape parameter $\nu$ one can see some bias. The bias can be reduced by simulating road profiles longer than $20 \mathrm{~km}$.

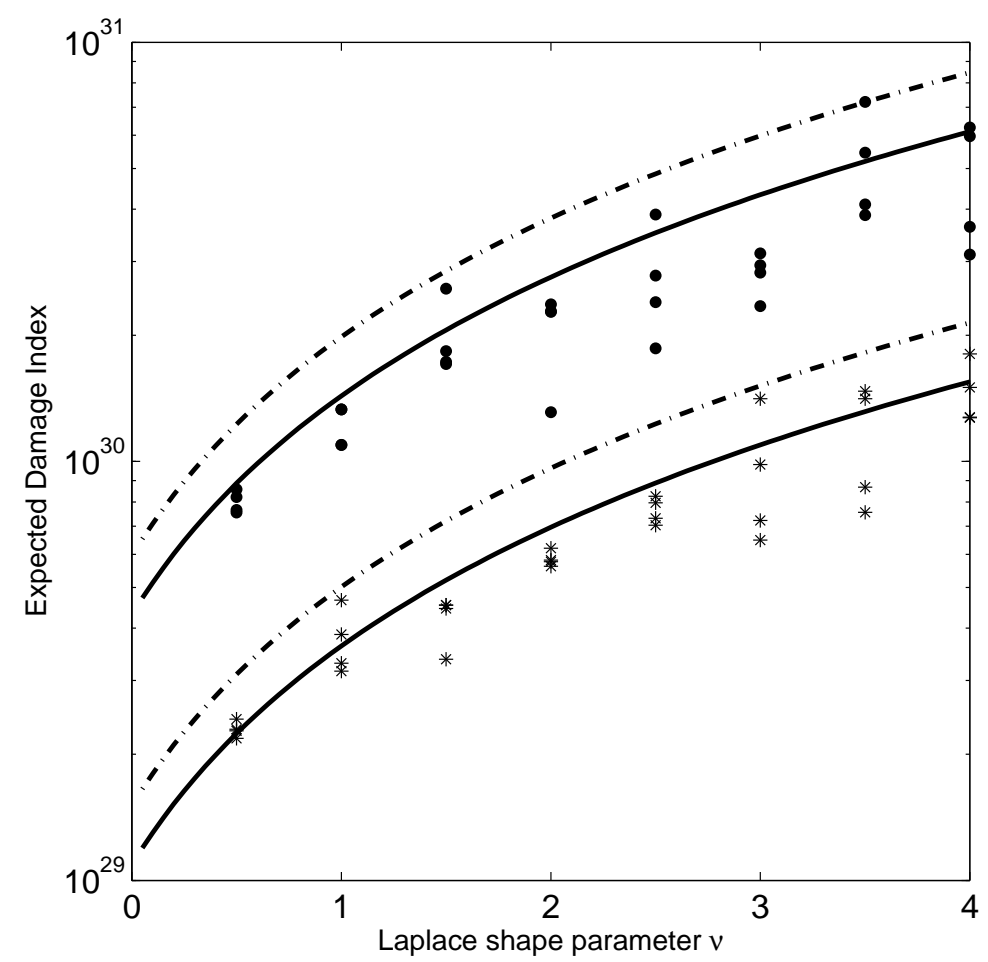

Figure 5: The expected damage indices $\mathrm{E}\left[D_{v}(k, 1, \nu)\right]$, approximation [30), for damage exponent $k=5$ and speeds $v=10,25 \mathrm{~m} / \mathrm{s}$ as function of parameter $\nu$. The solid lines are the approximation (30); the upper line is for $v=25 \mathrm{~m} / \mathrm{s}$ while the lower line is for $v=10 \mathrm{~m} / \mathrm{s}$. The dash-dotted lines are the narrow band bounds, Eq. (33). Again the upper line is for $v=25$ $\mathrm{m} / \mathrm{s}$ while the lower is for $v=10 \mathrm{~m} / \mathrm{s}$. The stars and dots are the observed damage indices from $20 \mathrm{~km}$ long simulations of the road profiles having $100 \mathrm{~m}$ long segments of constant variance. The dots are for $v=25 \mathrm{~m} / \mathrm{s}$ while the stars are for $v=10 \mathrm{~m} / \mathrm{s}$.

\subsection{The long-term damage index}

In Section 5.4 the non-stationary Laplace model for road surface roughness was introduced. Then in Section 5.6 means to estimate the parameters in the model with ISO spectrum were presented. The estimates require observations of the IRIs for road segments. Finally in the previous subsection we have demonstrated that the expected damage index can be accurately approximated, by means of formulas (30]33/35), for non-stationary Laplace model of road roughness having ISO spectrum. 
Since Eqs. (35) and (33) are given by explicit algebraic functions of model parameters these are very convenient for estimation of the long-term damage accumulation in a vehicle component. If the variability of parameters $\sigma^{2}$; the shape parameter $\nu$ in Laplace model and $v$ driving speed for a population of customers or a market is known and if the response can be described by means of a linear filter with an appropriate transfer function $H$ (here approximated by the quarter vehicle $H_{v}$, Eq. (10), then the expected long-term damage index $\mathrm{E}[D]$ can be approximated by means of the following integral

$$
\mathrm{E}[D] \approx \tilde{d}_{k} \int\left(\int v^{k / 2-1} f(v \mid \nu, \sigma) d v\right) \cdot\left(\nu \cdot \sigma^{2}\right)^{k / 2} \frac{\Gamma(k / 2+1 / \nu)}{\Gamma(1 / \nu)} f(\nu, \sigma) d \sigma d \nu .
$$

with the damage growth intensity $\tilde{d}_{k}=\mathrm{E}\left[D_{v_{0}}(k, 1,0)\right] / v_{0}^{k / 2-1}$, see Eq. 421) in Appendix $\mathrm{A}$ is easily available, see (Bengtsson and Rychlik, 2009). The density $f(\nu, \sigma)$ characterizes the encountered road quality, while the conditional density $f(v \mid \nu, \sigma)$ represents the driver behaviour.

\section{Validation of the Laplace-ISO model of road profiles}

A remaining important question is how well the Laplace-ISO model fits measured road profiles. In this section we shall validate the Laplace-ISO road profile model by studying the following issues:

1) Can the non-stationary Laplace model be used to reconstruct road profiles?

2) Can the ISO spectrum give sufficiently accurate approximations of road profiles?

3) Can the IRI be used to estimate the ISO spectrum?

4) What is the suitable length of segments with constant variance?

For the validation a data set of eight sections of roads with measured road profiles will be used. The eight selected sections represent different types of roads as well as different geographical locations. The lengths of the sections varies between 14 and 45 kilometres, see Table 2 second row. The measurements have been provided by Scania and were standardized to have zero mean and variance one. The signals are then filtered so that the low frequencies, with wavelength above 100 metres are removed. In the following the road profile will always mean the filtered road profile. The third row in the table contains estimates of standard deviations of the filtered signals, while the fourth row their kurtosis. One can see that the estimates of the kurtosis are significantly higher than 3 implying that road profiles should not be modelled as a stationary Gaussian processes.

The accuracy of the model will be validated by means of relative indices, i.e. fractions of the damage indices derived from a model and the observed indices, for various values of parameters the speed $v$ the damage exponent $k$ and length $L$ of constant variance segments. A relative index equal to one means that the damage index computed for the model is equal to the observed index in the measured profile.

\subsection{Laplace model with observed spectra}

In this section we demonstrate that the general non-stationary Laplace model can be used to describe the variability of the eight measured road profiles. In the model symmetrical kernels $g$ are used, which are estimated using Eq. (18) where $S_{0}(\Omega)$ is replaced by empirical spectra. 
Table 2: Rows 2-4; length, standard deviation and kurtosis of eight measured road elevations, respectively. In the fifth row are estimates of the Laplace shape parameter $\nu$ computed by means of Eq. 25); $\hat{\nu}=(\hat{\kappa}-3) / 3$.

\begin{tabular}{|c|c|c|c|c|c|c|c|c|}
\hline road number & 1 & 2 & 3 & 4 & 5 & 6 & 7 & 8 \\
\hline Length $[k m]$ & 32.2 & 13.7 & 37.0 & 44.3 & 44.8 & 23.1 & 14.5 & 39.5 \\
Standard deviation, $\hat{\sigma}$ & 0.34 & 0.41 & 0.37 & 0.29 & 0.38 & 0.28 & 0.37 & 0.38 \\
Kurtosis, $\hat{\kappa}$ & 4.23 & 5.49 & 8.62 & 4.88 & 6.05 & 6.55 & 3.79 & 5.31 \\
Shape parameter, $\hat{\nu}$ & 0.41 & 0.83 & 1.87 & 0.63 & 1.02 & 1.18 & 0.26 & 0.77 \\
\hline
\end{tabular}

The parameter $\sigma^{2}$ is estimated from the observed variance given in the third row of Table 2 The parameter $\nu$ has been estimated using Eq. 25), viz. $\hat{\nu}=(\hat{\kappa}-3) / 3$, see fifth row in the table. Note that this estimate of parameter $\nu$ are independent of $\sigma$ and $L$, the length of constant variance segments. Finally, in order to simulate the non-stationary Laplace model, also the length of segments of constant variance has to be chosen.

The accuracy of the Laplace model is validated by means of the following Monte Carlo study. Relative damages, fractions between simulated and observed damage indices, are used as measures of model accuracy. Three factors are considered; length of the constant variance segment on three levels $L=100,200,400 \mathrm{~m}$; damage exponent on three levels $k=3,4,5$; and speed on two levels $v=10,15 \mathrm{~m} / \mathrm{s}$. For each combination of factors and measured roads one Laplace road profile has been simulated, in total 144 road profiles. (The simulated profiles were of the same length as the corresponding measured profiles.) In Figure 6 three box plots are presented for relative damages, for $k=3,4,5$. (Each box plot is based on 48 reconstructed road profiles.) From the figure one can see that relative indices are close to one which means that damage indices computed for the non-stationary Laplace model agrees very well with the observed one for wide range of values of the considered factors. Medians of relative damage indices are about 1.2 indicating that Laplace model is slightly more damaging than the measured profiles.

\subsection{Laplace model with ISO spectrum}

In the previous section we have shown that the non-stationary Laplace model having observed spectrum reproduces the damage indices very well. In this section we turn to the second problem which is whether the simpler Laplace-ISO model could be used instead. In objective terms we shall look for eight sets of parameters $(C, \nu)$ such that the expected relative damage indices, denoted by $d(C, \nu, k, v)$, are close to one, say, in the interval [0.5, 2], for typical values of damage exponents $k$ and vehicle speeds $v$. The expected relative index is computed using Eq. (35), viz.

$$
d(C, \nu, k, v)=\frac{\mathrm{E}\left[D_{v}^{I S O}(k)\right]}{D_{v}^{o b s}(k)} \approx \frac{0.07093 e^{13.92 k}}{D_{v}^{o b s}(k)}\left(\frac{C}{C_{0}}\right)^{k / 2}\left(\frac{v}{v_{0}}\right)^{k / 2-1} \nu^{k / 2} \frac{\Gamma(k / 2+1 / \nu)}{\Gamma(1 / \nu)},
$$

where $C_{0}=14.4 \mathrm{~m}^{3}$ and $v_{0}=10 \mathrm{~m} / \mathrm{s}$.

First we check if one could simply replace the general kernels $g$ by the ISO kernels defined by $C=14.4 \sigma^{2}$, where $\sigma$ are taken from the third row in Table 2 Parameters $\nu$ are taken from the fifth row of the table. The resulting estimates $C$ and $\hat{\nu}$ are presented in the second and 


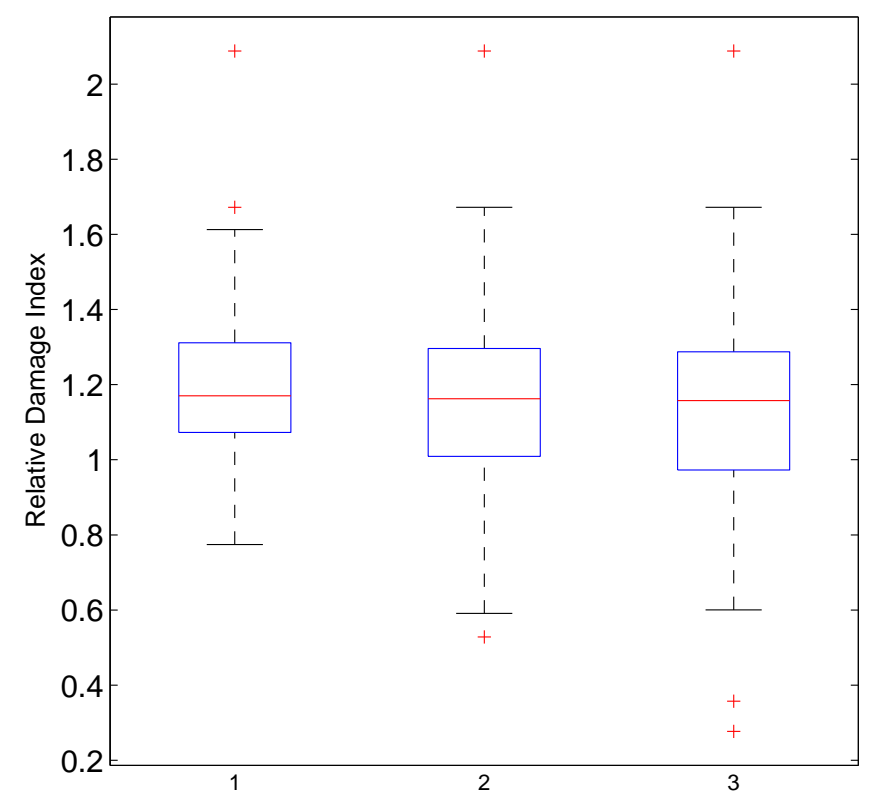

Figure 6: Three box plots of relative damages estimated for the general non-stationary Laplace model, for damage exponents $k=3,4,5$, respectively. One relative index is computed for each of the eight roads and combinations of the following factors $v=10,15 \mathrm{~m} / \mathrm{s}, L=100,200,400$ $\mathrm{m}$, i.e. each box plot is based on 48 relative indices.

third rows of Table 3 The relative indices $d(C, \nu, k, v)$ were computed for $v=10 \mathrm{~m} / \mathrm{s}$ and $k=3,5$ and presented in the fourth and fifth row of the table. One can see that the accuracy of this Laplace-ISO model is poor. The models are very conservative. In order to judge the roughness of the measured road profiles the logarithms of the observed damage indices $D_{v}^{o b s}(k)$, for $k=3,5$, are given in the last two rows in Table 3 One can see that the roads $6,7,8$ are smoother (less damaging) than the first five ones.

Table 3: Row 2; estimated parameter $C=14.4 \sigma^{2}$, where $\sigma$ are taken from the second row in Table 2 Rows 3-4; relative damage indices, Eq. (40). Rows 5-6; the logarithms of observed damage indices for $k=3,5$, respectively, for speed $v=10 \mathrm{~m} / \mathrm{s}$.

\begin{tabular}{|c|c|c|c|c|c|c|c|c|}
\hline road number & 1 & 2 & 3 & 4 & 5 & 6 & 7 & 8 \\
\hline$\hat{C}$ & 1.66 & 2.42 & 1.97 & 1.21 & 2.08 & 1.13 & 1.97 & 2.08 \\
$\hat{\nu}$ & 0.41 & 0.83 & 1.87 & 0.63 & 1.02 & 1.18 & 0.26 & 0.77 \\
\hline$d(C, \nu, 3,10)$ & 1.4 & 2.4 & 5.1 & 1.5 & 3.5 & 3.6 & 16.2 & 7.9 \\
$d(C, \nu, 5,10)$ & 0.5 & 3.0 & 12.2 & 0.7 & 6.2 & 6.0 & 44.5 & 23.4 \\
$\ln \left(D_{v}^{\text {obs }}(3)\right)$ & 35.7 & 35.8 & 35.0 & 35.2 & 35.3 & 34.3 & 33.4 & 34.4 \\
$\ln \left(D_{v}^{\text {obs }}(5)\right)$ & 62.9 & 62.5 & 61.3 & 62.0 & 61.5 & 60.2 & 58.6 & 60.0 \\
\hline
\end{tabular}

We conclude that replacing the general kernel by the ISO kernel does not lead to a useful Laplace-ISO model. However, this does not say that such a model does not exist. We shall next propose a "semi optimal" Laplace-ISO model by estimating $(C, \nu)$ employing Eqs. 37 38 
using the observed damage indices $D_{v}^{o b s}(2), D_{v}^{o b s}(4)$. Parameters $(C, \nu)$ estimated in this way will define Laplace-ISO models which have expected damage indices equal to the observed damage indices for $k=2,4$, see Eq. (35). Unfortunately, the parameters $C$ and $\nu$ vary with $v$. Obviously, one could estimate $C$ and $\nu$ by means of least square method and get estimates that are independent of $v$, however we will not pursuit this here and just average parameter values over the speeds. The resulting Laplace-ISO models are presented in Table 4 rows 2 and 3. As can be seen in rows 4-7 in Table 4 the derived Laplace-ISO models are sufficiently accurate proving that "useful" Laplace-ISO models are available for the studied roads.

Table 4: Row 2-3; estimated parameters $C, \nu$ employing Eqs. (37) 38). Rows 4-7; relative damage indices, Eq. (40) for $k=3,5$ and speeds $v=10,15 \mathrm{~m} / \mathrm{s}$.

\begin{tabular}{|c|c|c|c|c|c|c|c|c|}
\hline road number & 1 & 2 & 3 & 4 & 5 & 6 & 7 & 8 \\
\hline$\hat{C}$ & 1.25 & 1.87 & 0.88 & 1.14 & 1.30 & 0.62 & 0.43 & 0.73 \\
$\hat{\nu}$ & 1.88 & 0.68 & 1.36 & 1.22 & 0.37 & 0.59 & 0.32 & 0.39 \\
\hline$d(C, \nu, 3,10)$ & 1.25 & 1.54 & 1.40 & 1.54 & 1.46 & 1.28 & 1.68 & 1.49 \\
$d(C, \nu, 5,10)$ & 0.77 & 1.35 & 1.19 & 1.01 & 1.00 & 0.78 & 1.08 & 1.15 \\
$d(C, \nu, 3,15)$ & 1.02 & 0.85 & 1.01 & 0.93 & 0.94 & 1.02 & 0.85 & 0.88 \\
$d(C, \nu, 5,15)$ & 1.02 & 0.52 & 0.41 & 0.51 & 0.56 & 0.80 & 0.49 & 0.55 \\
\hline
\end{tabular}

However, in practice the damage indices $D_{v}^{o b s}(2), D_{v}^{o b s}(4)$ are not available and hence other means to estimate parameters $(C, \nu)$ are of interest. For many roads the sequence of IRI can be found in various databases and can be used to estimate the parameters by means of formulas 28 29]. In the following section this approach is validated by studying the relative damage indices.

\subsection{Estimating Laplace-ISO models from IRI sequences}

In many countries the sequences of IRI are collected and saved in databases. Therefore, reconstruction of the road profiles from IRI sequences is of practical interest in cases when the measured profiles are not available. It may not always be clear how the sequence has been estimated hence the accuracy of the reconstruction can be hard to judge. Here, for validation purposes, we will estimate the sequence of IRI from the measured data and use these to estimate the parameters $(C, \nu)$ in Laplace-ISO models. More precisely, the method consists of the following two steps:

(I) For a road segment of constant variance, length $L=200 \mathrm{~m}$, the spectrum of the golden car response is estimated and the second order spectral moment $\lambda_{2}$ is calculated. Then the IRI index is evaluated by means of Eq. (5). The reason for choosing Eq. (5) instead of more general formula (3) is that Eq. (5) is based on the assumption that the road profile is Gaussian which is also used in the construction of the Laplace model.

(II) The estimated sequence of IRI is then transformed into a sequence of $C_{j}$ by means of Eq. (28). Finally, the parameter $C$ is estimated by the average of $C_{j}$, while $\nu$ is estimated by the square of the coefficient of variation of $C_{j}$, see formula (29), or by fitting a gamma distribution to $C_{j} / C$. Here the second approach is used. 
The estimated parameters in the Laplace-ISO models, using the two steps procedure, are given in Table 5, rows 2-3. The accuracy of the models is investigated using the relative damage indices, Eq. (40), computed for $k=3,5$ and speeds $v=10,15 \mathrm{~m} / \mathrm{s}$. The indices are presented in Table 5, rows 4-7. Based on the reported values of the relative damage indices one must conclude that the accuracy of the models, estimated using IRI, are in general not as good as the accuracy of the "semi optimal" Laplace-ISO models presented in the previous section.

We conclude that the presented approach to estimate Laplace-ISO models from IRI sequences is useful for reconstruction of road profiles when measurements are not available. In the presented validation study only models for the road profiles $6-8$, which are not very damaging, give too conservative damage estimates, while for the other roads the estimated damage is almost within a factor 2. The statistical procedures presented here need further improvements. Particularly the following two problems need further investigations. The first one is the choice of the length of constant variance segment $L$. Here we meet a typical trade of situation; selecting shorter vales of $L$ will lead to higher statistical uncertainties of IRI values making the proposed estimates of $\nu$ biased (too large); choosing longer $L$ values may lead to violation of the assumption of constant variance in a segment. The second problem is the division of the long road segments into homogeneous parts to which the Laplace-ISO model could be fitted. In fact, the eight examined road profiles are not completely homogeneous.

Table 5: Rows 2-3; estimated $C, \nu$ by means of Eqs. (28) 29) with $C_{j}$ estimated from IRI using Eq. (9) with constant variance segments $L=200 \mathrm{~m}$. Rows 4-7; relative damage indices, Eq. (40) for $k=3,5$ and speeds $v=10,15 \mathrm{~m} / \mathrm{s}$.

\begin{tabular}{|c|c|c|c|c|c|c|c|c|}
\hline road number & 1 & 2 & 3 & 4 & 5 & 6 & 7 & 8 \\
\hline$\hat{C}$ & 1.41 & 1.87 & 1.37 & 1.62 & 1.74 & 1.06 & 0.97 & 1.12 \\
$\hat{\nu}$ & 0.52 & 0.59 & 0.84 & 0.54 & 0.46 & 0.52 & 0.38 & 0.51 \\
\hline$d(C, \nu, 3,10)$ & 1.13 & 1.51 & 2.44 & 2.22 & 2.33 & 2.85 & 5.84 & 2.93 \\
$d(C, \nu, 5,10)$ & 0.36 & 1.24 & 2.41 & 1.33 & 2.30 & 2.82 & 8.96 & 3.85 \\
$d(C, \nu, 3,15)$ & 0.92 & 0.83 & 1.76 & 1.35 & 1.50 & 2.27 & 2.95 & 1.74 \\
$d(C, \nu, 5,15)$ & 0.49 & 0.48 & 0.84 & 0.67 & 1.30 & 2.87 & 4.07 & 1.84 \\
\hline
\end{tabular}

\subsection{Influence on length of constant variance segments}

In the previous section we have used the expected damage index for the Laplace-ISO model to compare with the observed damage index. However, in practical applications often simulated road profiles are needed. Suppose that one has a $20 \mathrm{~km}$ long road profile and we choose the length of the constant variance segment to be $500 \mathrm{~m}$. Then there will be only 40 random variances in the signal, compared to 200 when $L=100$. Since chances to get very high variance is much higher in the second case and one has higher frequency of transients when variance is changing values we expect that when $L=500$ the damage index will be smaller compared to the index for $L=100$. This will be illustrated in the following Monte Carlo study.

Using Laplace-ISO models, estimated from the IRI sequences and given in Table 5, 10 road profiles will be simulated of total length of $249.2 \mathrm{~km}$ for three values of parameter $L, 100,200$ 
and $500 \mathrm{~m}$, i.e. in total 30 road profiles. Note that each simulated road surface consists of 8 shorter segments having different values of parameters. The damage index will be then estimated for each of the $249 \mathrm{~km}$ long simulations and scaled by the observed damage index. The ratio will be called accumulated relative damage with value one if the damage index in the simulated road profile is equal to the measured damage index. The result of the simulation study is presented in Table6. One can see that the damage varies considerably with the chosen length $L$ demonstrating the importance of proper selection of parameter $L$.

Table 6: Mean, minimum and maximum values for 10 accumulated relative damages, for the Laplace-ISO models for the eight road sections with parameters given in Table 5 Three different values of the length $L$ has been used in the simulation algorithm.

\begin{tabular}{|c|c|c|c|c|c|c|c|c|c|c|c|c|}
\hline \multirow{3}{*}{ Model } & \multicolumn{5}{|c|}{ Speed $10 \mathrm{~m} / \mathrm{s}$} & \multicolumn{5}{c|}{ Speed $15 \mathrm{~m} / \mathrm{s}$} \\
\cline { 2 - 14 } & \multicolumn{3}{|c|}{$\mathrm{k}=3$} & \multicolumn{3}{|c|}{$\mathrm{k}=5$} & \multicolumn{3}{c|}{$\mathrm{k}=3$} & \multicolumn{3}{c|}{$\mathrm{k}=5$} \\
\cline { 2 - 14 } & mean & min & max & mean & min & max & mean & min & max & mean & min & max \\
\hline$L=500 \mathrm{~m}$ & 1.19 & 0.91 & 1.38 & 0.59 & 0.30 & 0.83 & 0.81 & 0.68 & 0.96 & 0.34 & 0.22 & 0.52 \\
$L=200 \mathrm{~m}$ & 1.86 & 1.81 & 1.93 & 1.09 & 1.00 & 1.23 & 1.19 & 1.09 & 1.29 & 0.58 & 0.49 & 0.70 \\
$L=100 \mathrm{~m}$ & 3.28 & 3.00 & 3.41 & 3.04 & 2.57 & 3.36 & 2.09 & 2.01 & 2.17 & 1.57 & 1.39 & 1.80 \\
\hline
\end{tabular}

\section{Conclusions}

The main goal has been to find a statistical model for road profiles, which can be estimated from a sequence of IRI measurements. The road profile can then be stochastically reconstructed. When measured road profiles are not available, but only condensed roughness data in the form of IRI values or roughness coefficients, a simple statistical model for the road profile is needed in order to be able to estimate the model parameters. However, the model should still be useful for durability applications. For this purpose, the Gaussian model has been found to be too simple, see e.g. (Bogsiö, 2007), since it can not correctly capture the variability of the roughness. For our setup we have found that the non-stationary Laplace model, (Bogsjö et al., 2012), with ISO spectrum, (ISO 8608, 1995), is simple enough but still useful for durability evaluations. It can be interpreted as a Gaussian process where the local variance is randomly varying according to a gamma distribution. The length of constant variance segments is predefined, and for road profiles typically one or some hundred metres. The non-stationary Laplace process can be modelled by two parameters, either by its variance and kurtosis or equivalently by its mean roughness and Laplace shape parameter.

A practically important theoretical finding is that the expected damage due to a Laplace road with ISO spectrum, can be approximated by an explicit algebraic expression, see Eq. (35). The formula depends on the damage exponent $k$, the speed $v$, the mean roughness coefficient $C$, and the Laplace shape parameter $\nu$. The first three factors corresponds to the damage due to a Gaussian model, while the last factor is a correction for the Laplace model, depending on the Laplace shape parameter $\nu$. The approximation has been validated by simulating Laplace roads, see Figure 5 .

An important question is how well the Laplace-ISO model fits measured road profiles. Therefore, a validation study was conducted, where a data set of measured road profiles were 
used. The eight road sections represent different types of roads as well as different geographical locations. The conclusions of the study can be summarized as

1. We have demonstrated that the non-stationary Laplace model having observed spectrum reproduces the damage indices very well.

2. We investigated whether the simpler Laplace-ISO model could be used instead. Simply replacing the observed spectrum by the an estimated ISO spectrum gave unsatisfactory accuracy. However, by estimating the parameters from observed damage values, a "useful" Laplace-ISO model was found for the studied roads.

3. We found that the presented approach to estimate Laplace-ISO models from IRI sequences is useful for reconstruction of road profiles when profile measurements are not available.

Some of measured road profiles are not statistically homogeneous and in order to improve a fit of Laplace modes to data one could consider to split it in shorter segments in which homogeneity is more likely, e.g. $5 \mathrm{~km}$ long segments. This would result in larger set of estimated models which would allow to study the long term distribution for the parameters $C$ and $\nu$ in the data and then to validate Eq. 39]. However, these investigations are outside of the scope of the present study and will be conducted in the future.

There are several advantages to use the Laplace road profile model with ISO spectrum

- a small number of parameters are needed to define it (the roughness coefficient, $C$, the Laplace shape parameter, $\nu$, and the length of constant variance road segment, $L$ ),

- the parameters $C$ and $\nu$ can be estimated from the sequence of IRI, see Eq. (26) which is often available, and

- the expected damage of a response of a vehicle, modelled by a linear filter having Laplace-ISO road as an input, can be accurately approximated by an explicit formula depending only on the Laplace parameters, $(C, \nu)$, the damage exponent, $k$, and the speed $v$, see e.g. Eq. (35).

The last property is particularly convenient for sensitivity studies since lengthy simulations can be avoided. It can also be used for estimation of Laplace parameters and for classification purposes.

\section{Acknowledgments}

This work is partially supported by a research project financed by DAF, Daimler, MAN, Scania and Volvo. Further, we are thankful to Scania for supplying us with road profile data.

\section{References}

P. Andrén. Power spectral density approximations of longitudinal road profiles. Int. J. Vehicle Design, 40:2-14, 2006.

J. S. Bendat. Probability functions for random responses: Prediction of peaks, fatigue damage and catastrophic failures. Technical report, NASA, 1964. 
A. K. Bengtsson and I. Rychlik. Uncertainty in fatigue life prediction of structures subject to Gaussian loads. Probabilistic Engineering Mechanics, 24:224-235, 2009.

K. Bogsjö, K. Podgorski, and I. Rychlik. Models for road surface roughness. Vehicle System Dynamics, 50:725-747, 2012.

K. Bogsjö. Road Profile Statistics Relevant for Vehicle Fatigue. PhD thesis, Mathematical Statistics, Lund University, 2007.

K. Bogsjö and I. Rychlik. Vehicle fatigue damage caused by road irregularities. Fatigue \& Fracture of Engineering Materials \& Structures, 32:391-402, 2009.

P. A. Brodtkorb, P. Johannesson, G. Lindgren, I. Rychlik, J. Rydén, and E. Sjö. WAFO a Matlab toolbox for analysis of random waves and loads. In Proceedings of the 10th International Offshore and Polar Engineering conference, Seattle, volume III, pages 343$350,2000$.

B. Bruscella, V. Rouillard, and M. Sek. Analysis of road surfaces profiles. ASCE Journal of Transportation Engineering, 125:55-59, 1999.

D. Charles. Derivation of environment descriptions and test severities from measured road transportation data. Journal of the IES, 36:37-42, 1993.

C. J. Dodds and J. D. Robson. The description of road surface roughness. Journal of Sound and Vibration, 31:175-183, 1973.

T. D. Gillespie, M. W. Sayers, and C. A. V. Queiroz. The international road roughness experiment: Establishing correlation and calibration standard for measurement. Technical Report No. 45, The World Bank, 1986.

A. González, E. J. O'brie, Y.-Y. Li, and K. Cashell. The use of vehicle acceleration measurements to estimate road roughness. Vehicle System Dynamics, 46:483-499, 2008.

J. G. Howe, J. P. Chrstos, R. W. Allen, T. T. Myers, D. Lee, C.-Y. Liang, D. J. Gorsich, and A. A. Reid. Quarter car model stress analysis for terrain/road profile ratings. Int. J. Vehicle Design, 36:248-269, 2004.

ISO 8608. Mechanical vibration - road surface profiles - reporting of measured data, ISO 8608:1995(E). International Organization for Standardization, ISO, 1995.

S. Kotz, T. J. Kozubowski, and K. Podgórski. The Laplace Distribution and Generalizations: A Revisit with Applications to Communications, Economics, Engineering and Finance. Birkhaüser, Boston, 2001.

O. Kropáč and P. Múčka. Non-standard longitudinal profiles of roads and indicators for their characterization. Int. J. Vehicle Design, 36:149-172, 2004.

O. Kropáč and P. Múčka. Indicators of longitudinal road unevenness and their mutual relationships. Road Materials and Pavement Design, 8:523-549, 2007.

R. P. La Barre, R. T. Forbes, and S. Andrew. The measurement and analysis of road surface roughness. Report 1970/5, Motor Industry Research Association, 1969.

M. A. Miner. Cumulative damage in fatigue. Journal of Applied Mechanics, 12:A159-A164, 1945. 
H. M. Ngwangwa, P. S. Heyns, F. J. J. Labuschagne, and G. K. Kululanga. Reconstruction of road defects and road roughness classification using vehicle responses with artificial neural networks simulation. Journal of Terramechanics, 47:97-111, 2010.

A. Palmgren. Die Lebensdauer von Kugellagern. Zeitschrift des Vereins Deutscher Ingenieure, 68:339-341, 1924. In German.

V. Rouillard. Using predicted ride quality to characterise pavement roughness. Int. J. Vehicle Design, 36:116-131, 2004.

V. Rouillard. Decomposing pavement surface profiles into a Gaussian sequence. Int. J. Vehicle Systems Modelling and Testing, 4:288-305, 2009.

I. Rychlik. A new definition of the rainflow cycle counting method. International Journal of Fatigue, 9:119-121, 1987.

I. Rychlik. On the 'narrow-band' approximation for expected fatigue damage. Probabilistic Engineering Mechanics, 8:1-4, 1993.

M. Shinozuka. Simulation of multivariate and multidimensional random processes. The Journal of the Acoustical Society of America, 49:357-368, 1971.

WAFO Group. WAFO - a Matlab toolbox for analysis of random waves and loads, tutorial for WAFO 2.5. Mathematical Statistics, Lund University, 2011a.

WAFO Group. WAFO - a Matlab Toolbox for Analysis of Random Waves and Loads, Version 2.5, 07-Feb-2011. Mathematical Statistics, Lund University, $2011 \mathrm{~b}$.

Web: http://www.maths.1th.se/matstat/wafo/(Accessed 12 August 2012).

\section{Appendix}

\section{A Sketch of derivation of approximation (30)}

We assume that the rainflow damage can be computed for a response observed for each of the segments with constant variance separately and then added. (This is a reasonable approximation if the mean response is constant for longer period of time.) Under this assumption

$$
\mathrm{E}\left[D_{v}(k, 1, \nu)\right]=\mathrm{E}\left[D_{v}(k, 1,0)\right] \mathrm{E}\left[R^{k / 2}\right],
$$

by independence of the factors $R_{j}$ and Gaussianity of road roughness. Next

$$
\mathrm{E}\left[R^{k / 2}\right]=\int_{0}^{\infty} r^{k / 2} f_{R}(r) d r=\nu^{k / 2} \frac{\Gamma(k / 2+1 / \nu)}{\Gamma(1 / \nu)} .
$$

Finally, for a Gaussian model, one can show that for any pair of nonzero speeds $v, v_{0}$ one has that

$$
\mathrm{E}\left[D_{v}(k, 1,0)\right] / v^{k / 2-1}=\mathrm{E}\left[D_{v_{0}}(k, 1,0)\right] / v_{0}^{k / 2-1},
$$

which shows Eq. (30). 
For simplicity Eq. (41) will be demonstrated only for Shinozuka method, (Shinozuka, 1971), to simulate Gaussian processes. Consider the linear response $Y_{v}(x)$ to Gaussian road profile having standard ISO spectrum (roughness coefficient $C=14.4$ );

$$
Y_{v}(x)=\sqrt{C} \sum_{i=1}^{n} \Omega_{i}^{-1}\left|H_{v}\left(\Omega_{i}\right)\right| \sqrt{\Delta \Omega} \cos \left(\Omega_{i} x+\phi_{i}\right) . \quad 0 \leq x \leq L .
$$

Employing the relation $\omega_{i}=\Omega_{i} v$ and that $H_{v}(\Omega)=H(\Omega v)$ then, with $t=x / v$, the last equation can be written as follows

$$
Y_{v}(x)=\sqrt{v} \sqrt{C} \sum_{i=1}^{n} \omega_{i}^{-1}\left|H\left(\omega_{i}\right)\right| \sqrt{\Delta \omega} \cos \left(\omega_{i} t+\phi_{i}\right)=\sqrt{v} \tilde{Y}(t), \quad 0 \leq t \leq L / v .
$$

Denote by $\tilde{d}_{k}$ damage growth intensity in $\tilde{Y}(t)$ then

$$
\mathrm{E}\left[D_{v}(k, 1,0)\right]=\frac{1}{L} \cdot\left(\frac{L}{v} v^{k / 2} \tilde{d}_{k}\right),
$$

and hence $\mathrm{E}\left[D_{v}(k, 1,0)\right] / v^{k / 2-1}=\tilde{d}_{k}$ independently of $v$ proving the relation (41).

\section{B MATLAB code for model simulation}

For readers convenience we present the MATLAB codes used to simulate responses to the Gaussian and the non-stationary Laplace models for the road profile. From a sequence of IRI, code for estimation of the Gaussian and non-stationary Laplace models is given, as well as directions for simulating the non-stationary Gaussian model. Finally, code for calculation of the expected damage is given.

In the code some functions from the WAFO (Brodtkorb et al,, 2000; WAFO Group, 2011a) toolbox are used, which can be downloaded free of charge, (WAFO Group, 2011b). The statistical functions rndnorm and rndgam are also available in the MATLAB statistics toolbox through normrnd and gamrnd. Note that WAFO also contains functions to find rainflow ranges used to estimate fatigue damage.

The length of the simulated function will be $5 \mathrm{~km}$ and the sampling interval $5 \mathrm{~cm}$. The following code can be used to compute the spectrum.

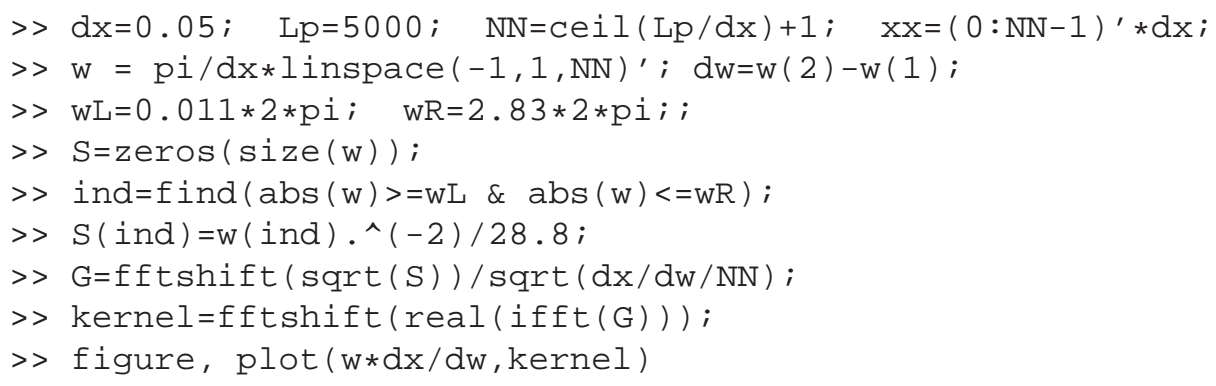

The kernel $g(x)$ is introduced through its Fourier transform $G(\Omega)=\mathcal{F} g(\Omega)$. We use a normalized $g(x)$ so that the integral $\int g(x)^{2} \mathrm{~d} x=1$, and hence we need an additional parameter $\sigma$, i.e. the standard deviation of the road, in the code denoted by SD. If the load is Gaussian then $\sigma$ is constant for whole length $L p$ and need to be estimated from the signal. This is not a trivial problem since the true spectrum often differs from the ISO one, but we do not go into details in this issues.

The transfer function $H_{v}(\Omega)$ given by Eq. (10) is computed by 


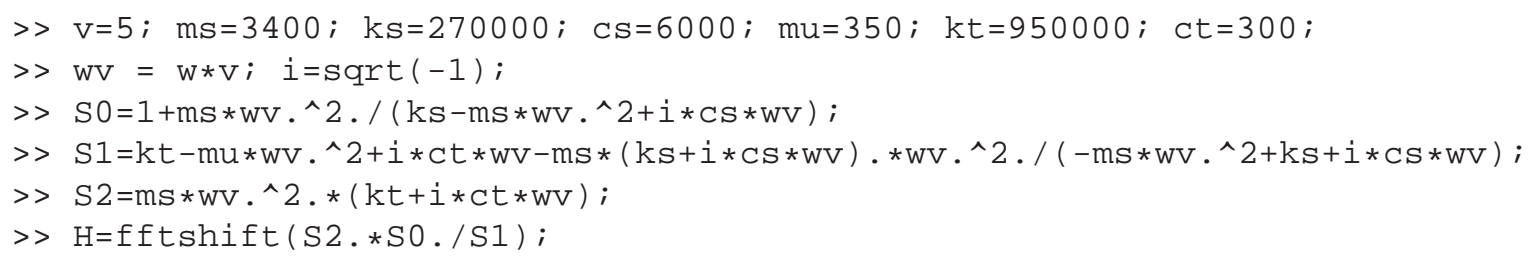

We turn now to simulation of Gaussian and Laplace models.

\section{B.1 Gaussian model}

First a Gaussian white noise process InpG is generated, then the road profile and quarter vehicle response $z G$ and $y G$, respectively, are computed by means of FFT.

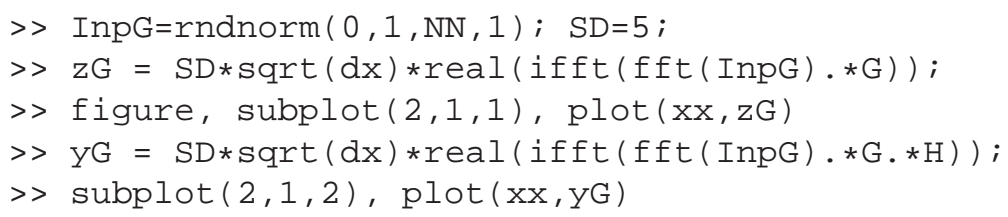

\section{B.2 Non-stationary Laplace model}

In the Laplace model it is assumed that parameter $\sigma$ is constant for a short segment of a road, here 200 metres. First the shape parameter $\nu$, see Eq. (25), is computed from road profile kurtosis kurt, here set to 9 . This determines the gamma distributed random variances $R$. Then the modulation process mod is evaluated and finally road elevation $z L$ and quarter vehicle response $\mathrm{yL}$ are computed.

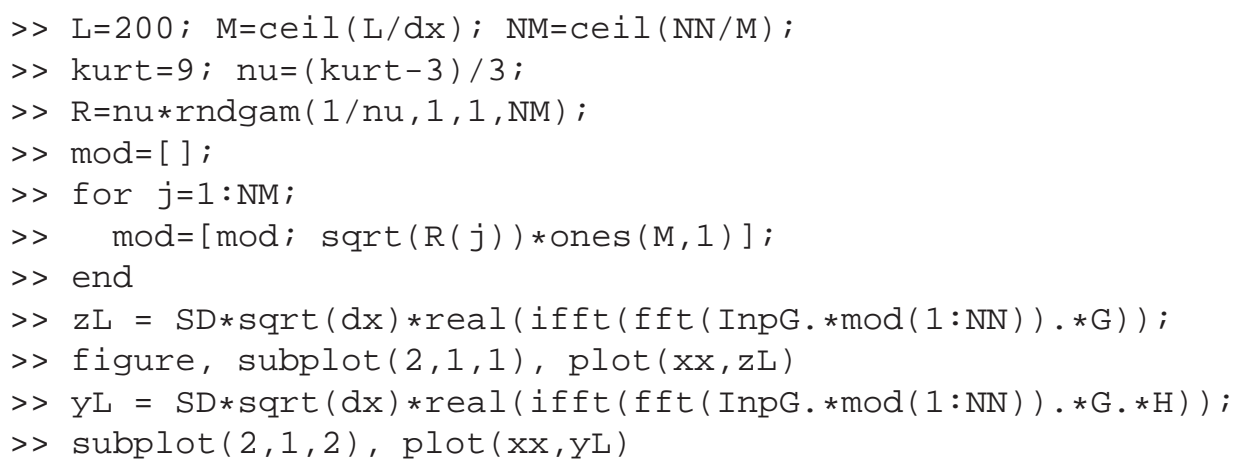

Note that in the code the same sample of a Gaussian white noise InpG has been used to generate the Gaussian and non-stationary Laplace models of the road profile. This is done to facilitate visual comparison of the simulated records.

\section{B.3 Estimation of non-stationary Laplace model}

Here we assume that from some database the sequence of IRI are available sampled also at 200 metres. The sequence is saved in a vector IRI.

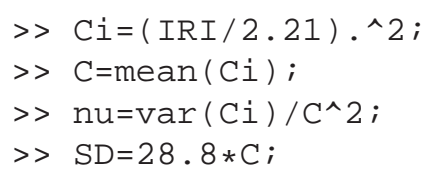


The estimated parameters $\mathrm{C}$ and nu of the non-stationary Laplace model can then be used for simulating profiles. Note that if the simulated gamma variables $\mathrm{R}$ are replaced by a corresponding vector of observed normalized variances $\mathrm{R}=\mathrm{Ci} / \mathrm{C}$, the same simulation code can be used for simulating a non-stationary Gaussian profile.

\section{B.4 Expected damage}

Here we check the result of Eq. (30); compare Figure 5] The following code simulates Laplace roads with different shape parameters $\nu$ and calculates the observed the fatigue damage index, which is compared with the theoretical formula $[30$.

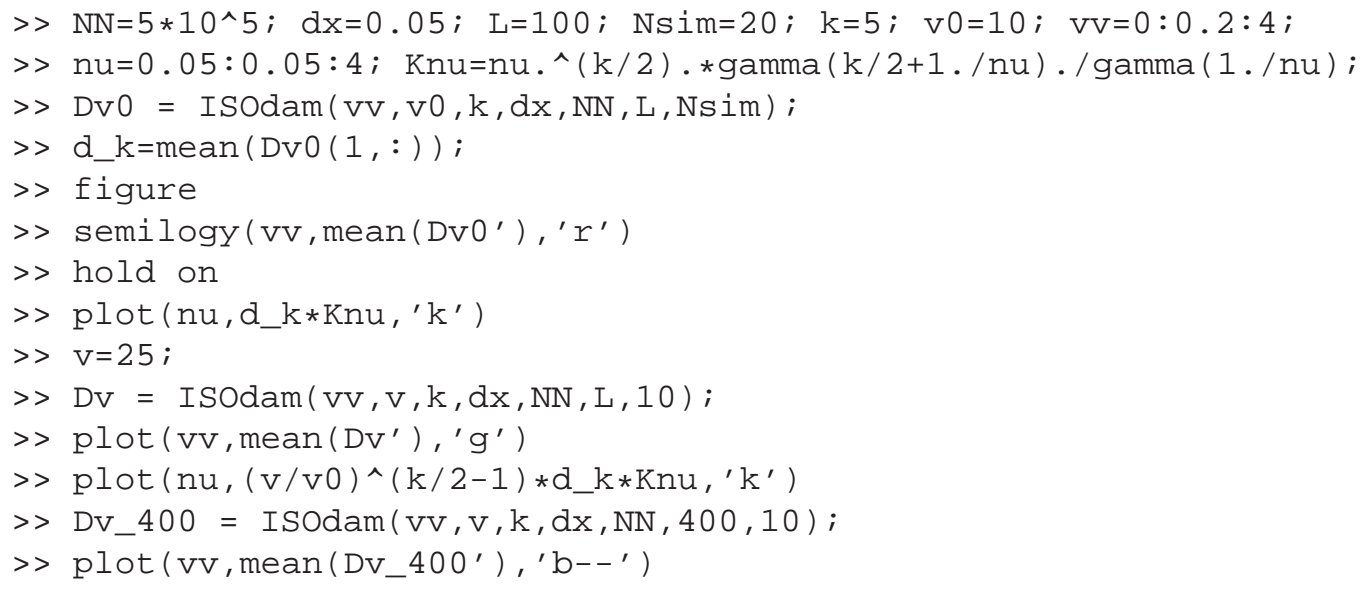

The code needs two functions called ISOdam.m, simulating Laplace roads and calculating damage

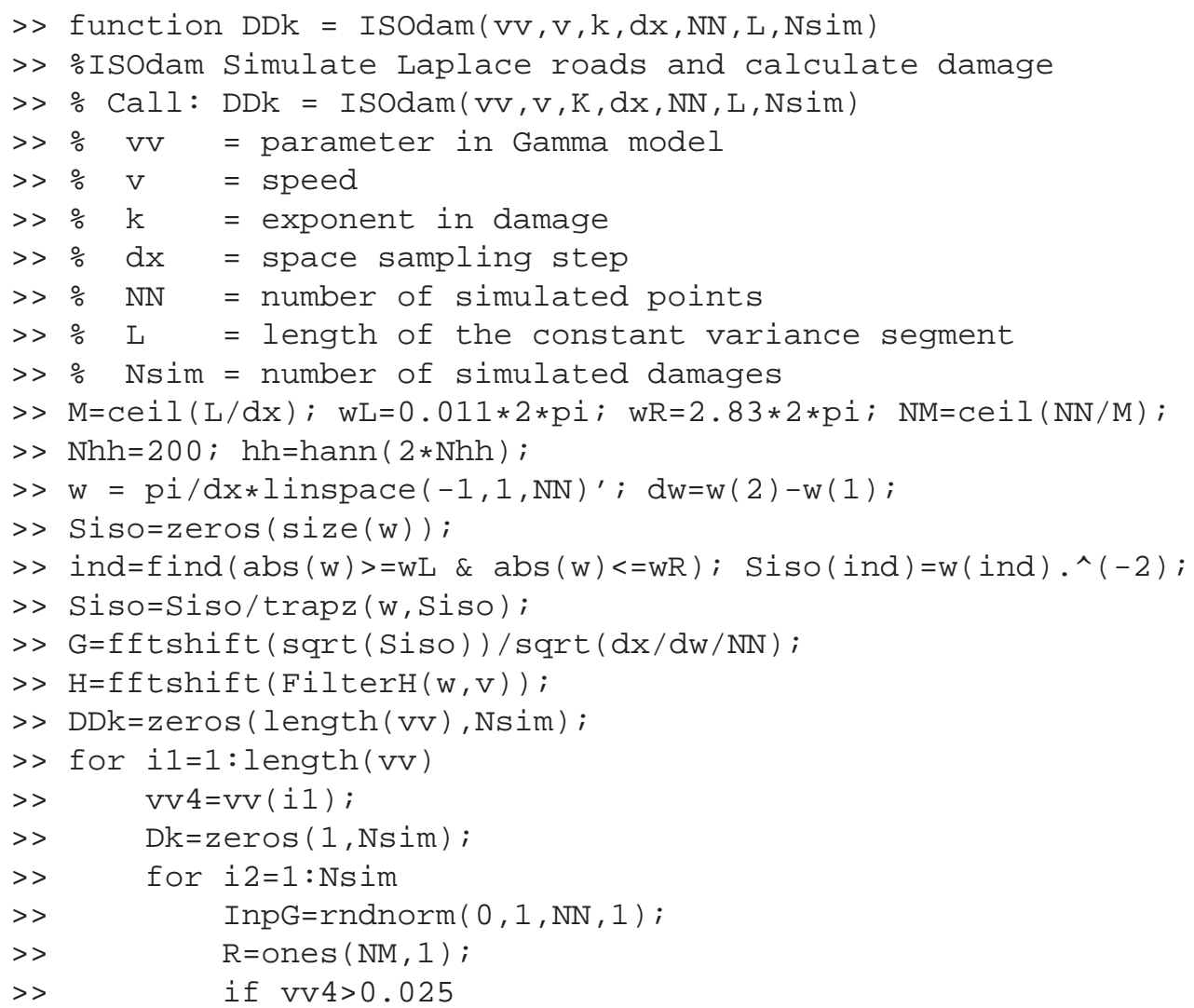




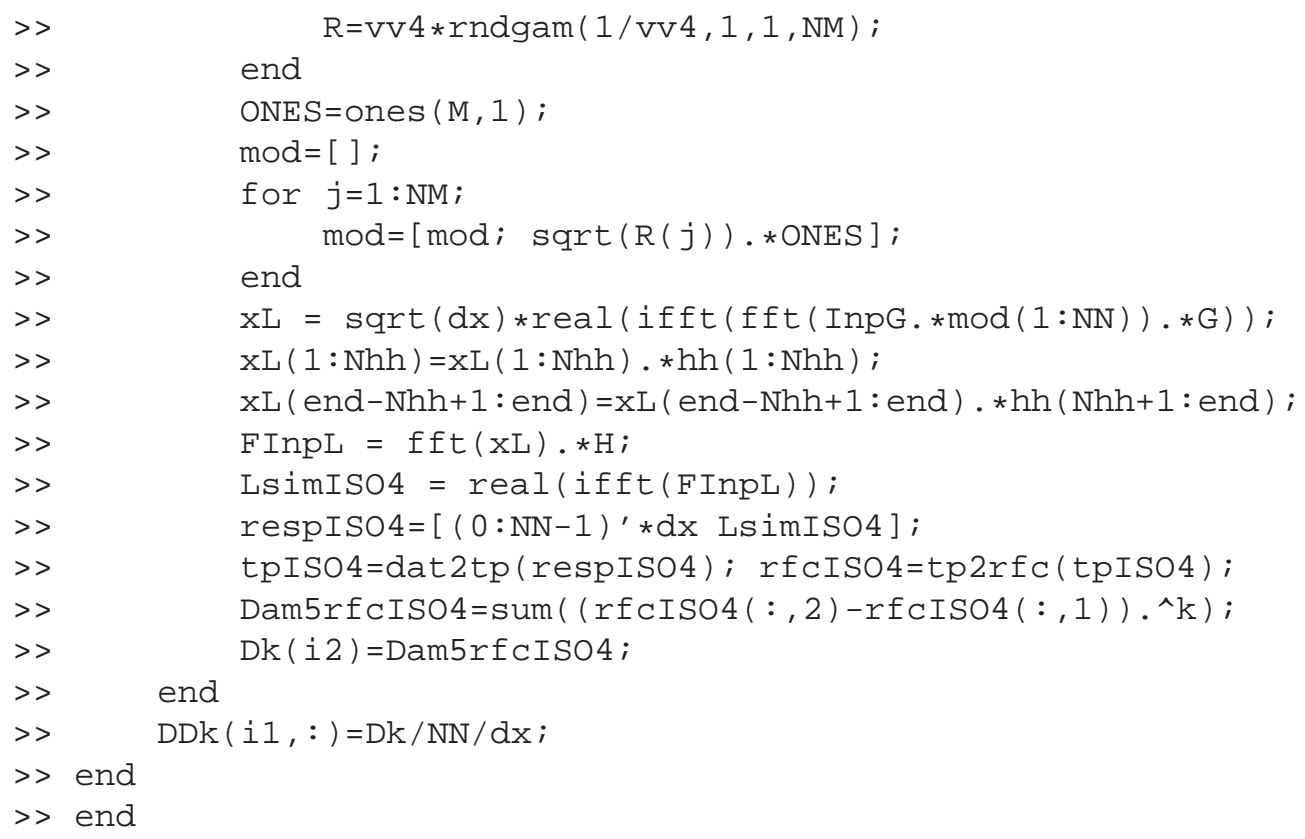

and FilterH.m defining the transfer function.

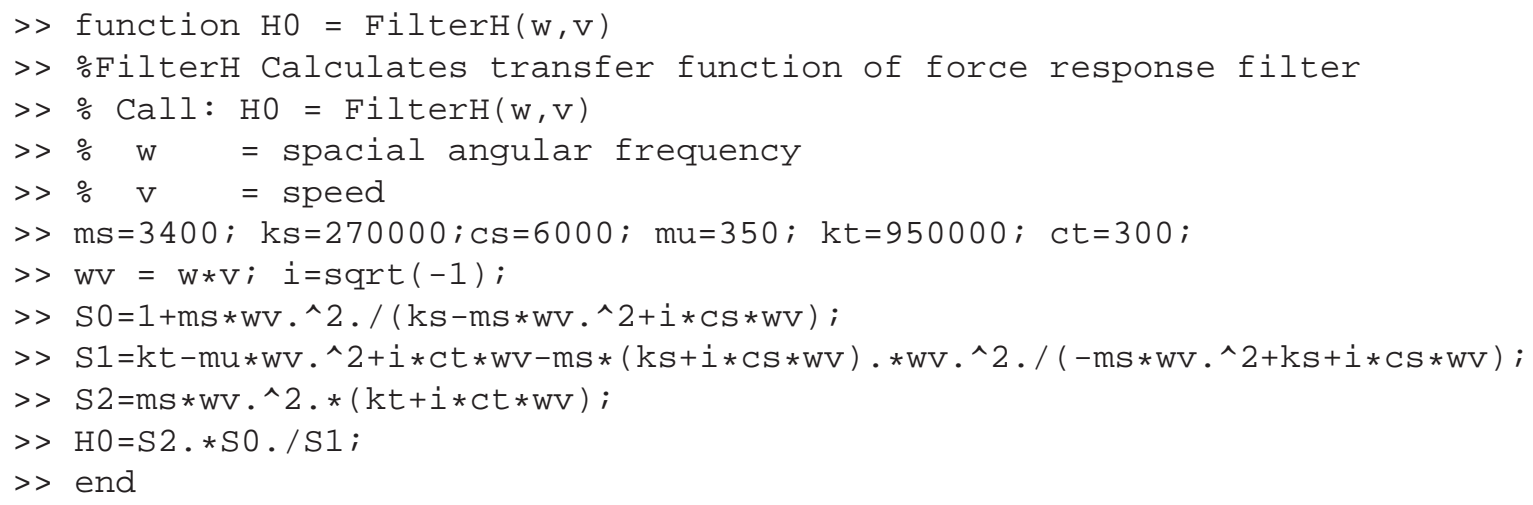




\section{SP Sveriges Tekniska Forskningsinstitut}

Vi arbetar med innovation och värdeskapande teknikutveckling. Genom att vi har Sveriges bredaste och mest kvalificerade resurser för teknisk utvärdering, mätteknik, forskning och utveckling har vi stor betydelse för näringslivets konkurrenskraft och hållbara utveckling. Vår forskning sker i nära samarbete med universitet och högskolor och bland våra cirka 10000 kunder finns allt från nytänkande småföretag till internationella koncerner.

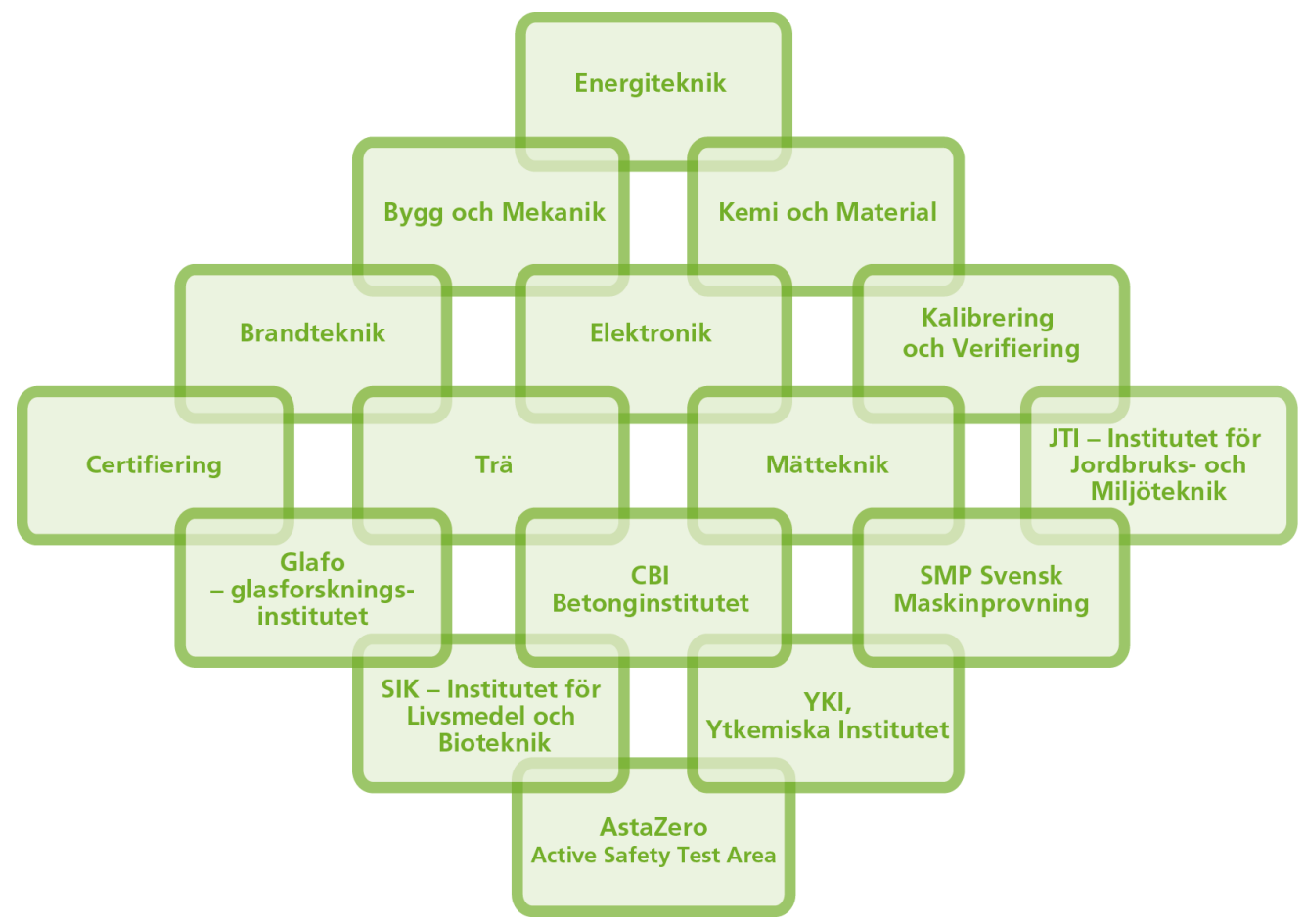

SP Sveriges Tekniska Forskningsinstitut Box 857, 50115 BORĂS

Telefon: 010-516 50 00, Telefax: 033-13 5502

E-post: info@sp.se, Internet: www.sp.se

www.sp.se

Mer information om SP:s publikationer: www.sp.se/publ
SP Bygg \& Mekanik

SP Rapport 2012:43

ISBN 978-91-87017-61-2

ISSN 0284-5172 